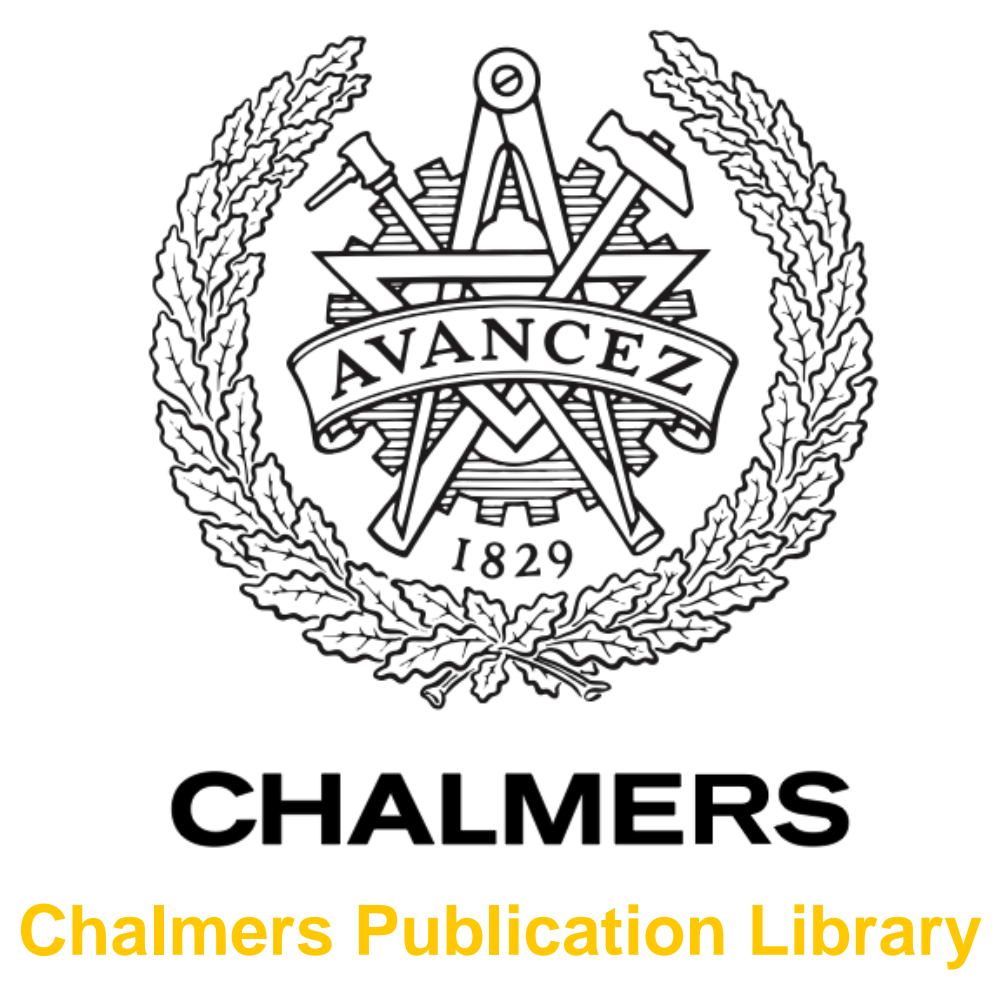

Parametric Analysis of a Large-scale Cycloidal Rotor in Hovering Conditions

This document has been downloaded from Chalmers Publication Library $(\mathrm{CPL})$. It is the author's version of a work that was accepted for publication in:

Journal of Aerospace Engineering (ISSN: 0893-1321)

Citation for the published paper:

Xisto, C. ; Leger, J. ; Páscoa, J. et al. (2016) "Parametric Analysis of a Large-scale Cycloidal Rotor in Hovering Conditions". Journal of Aerospace Engineering

http://dx.doi.org/10.1061/(ASCE)AS.1943-

5525.0000658

Downloaded from: http://publications.lib.chalmers.se/publication/235793

Notice: Changes introduced as a result of publishing processes such as copy-editing and formatting may not be reflected in this document. For a definitive version of this work, please refer to the published source. Please note that access to the published version might require a subscription. 


\title{
PARAMETRIC ANALYSIS OF A LARGE-SCALE CYCLOIDAL ROTOR IN HOVERING CONDITIONS
}

\author{
Carlos M. Xisto (corresponding author) $)^{1}$, J. A. Leger ${ }^{2}$, J. C. Páscoa ${ }^{3}$, \\ L. Gagnon ${ }^{4}$, P. Masarati ${ }^{5}$, \\ D. Angeli ${ }^{6}$ and A. Dumas ${ }^{7}$
}

\section{ABSTRACT}

In this work, four key design parameters of cycloidal rotors, namely the airfoil section; the number of blades; the chord-to-radius ratio; and the pitching axis location, are addressed. The four parameters, which have a strong effect on the rotor aerodynamic efficiency are analyzed with an analytical model and a numerical approach. The numerical method is based on a finite-volume discretization of two-dimensional Unsteady Reynolds Averaged Navier-Stokes equations on a multiple sliding mesh, are proposed and validated against experimental data. A parametric analysis is then carried out considering a large-scale cyclogyro, suitable for payloads above $100 \mathrm{~kg}$, in hovering conditions. Results demonstrate that the airfoil thickness significantly affects the rotor performance; such a result is partly in contrast with previous findings for small- and micro-scale configurations. Moreover, it will be shown that increasing the number of blades could result in a decrease of the rotor efficiency. The effect of chord-to-radius will demonstrate that values of around 0.5 result in higher efficiency. Finally it is found out that for these large systems, in contrast with micro-scale cyclogyros, the generated thrust increases as the pitching axis is located away from the leading edge, up to $35 \%$ of chord length. Further the shortcomings of using simplified analytical tools in the prediction of thrust and power in non-ideal flow conditions will be highlighted and discussed.

Keywords: Cycloidal Rotor, Cyclogyro, Computational Fluid Dynamics, Parametric study, Analytical Aerodynamics.

\section{INTRODUCTION}

A cycloidal rotor, also known as cyclogyro or cyclocopter, is a horizontal-axis rotary-wing machine capable of mechanically control the pitching schedule and pitching amplitude of the blades (Nakonechny 1961; Foshag and Boehler 1969; Yun et al. 2005). Such a mechanical system allows for the generation of a net aerodynamic force (perpendicular to the rotation axis), which can be easily controlled in terms of magnitude and direction (Kirsten 1928; Nozaki et al. 2009). This important feature makes the cyclogyro an attractive propulsion system for a wide range of applications, since

\footnotetext{
${ }^{1}$ Postdoctoral Researcher, Division of Fluid Dynamics, Department of Applied Mechanics, Chalmers University of Technology, Gothenburg, 41296, Sweden. Email: carlos.xisto@chalmers.se

${ }^{2}$ Ph.D. Student, Departamento de Eng. Electromecânica, Universidade da Beira Interior, Covilhã, 6201-001, Portugal. C-MAST: Center for Mechanical and Aerospace Science and Technology, Research Unit No 151. Email: leger_mec@hotmail.com

${ }^{3}$ Assistant Professor, Departamento de Eng. Electromecânica, Universidade da Beira Interior, Covilhã, 6201-001, Portugal. C-MAST: Center for Mechanical and Aerospace Science and Technology, Research Unit No 151. Email: pascoa@ubi.pt

${ }^{4}$ Research Associate, Dip. di Scienze e Tecnologie Aerospaziali, Politecnico di Milano, 20156 Milano, Italia. Email: louis.gagnon@polimi.it

${ }^{5}$ Associate Professor, Dip. di Scienze e Tecnologie Aerospaziali, Politecnico di Milano, 20156 Milano, Italia. Email: pierangelo.masarati@polimi.it

${ }^{6}$ Assistant Professor, Dip. di Scienze e Metodi dell'Ingeneria, Universita di Modena e Reggio Emilia, 42122, Reggio Emilia, Italia. Email: diego.angeli@unimore.it

${ }^{7}$ Professore Ordinario, Dip. di Scienze e Metodi dell'Ingeneria, Universita di Modena e Reggio Emilia, 42122, Reggio Emilia, Italia. Email: antonio.dumas@unimore.it
} 
it can improve the maneuverability of the aircraft for several flight conditions, e.g. VTOL (Vertical Take-Off and Landing) and STOL (Short Take-Off and Landing), hovering and forward flight conditions (Siegel et al. 2007; Yu et al. 2006; Ilieva et al. 2014; Trancossi et al. 2014). Furthermore, these machines normally operate at a lower noise level than conventional helicopters, and it was demonstrated that an optimized cyclogyro can generate higher thrust per power input (higher power loading) when compared with a conventional screw propeller, operating at a similar disk load (thrust generated per unit area on the rotor actuator disk) (Boschma 2001; Gibbens 2003; Benedict et al. 2013). A three-dimensional representation of a cycloidal rotor and a possible incorporation into an aircraft are illustrated in Fig. 1-a) and b), respectively. For the compound helicopter concept, exemplified in Fig. 1-b), lift is produced by the main rotor, and two cyclogyros are included to provide anti-torque, yaw and roll control and to extend the subsonic flight regime of the aircraft. In this configuration, the cyclogyros take over the task of producing thrust, relieving the main rotor, which is requested a lower amount of thrust. As a consequence, stall on the retreating blade is pushed towards higher forward flight speeds.

The aerodynamic efficiency of a cyclogyro depends on several design parameters, e.g. number of blades, airfoil section, blade flexibility, blade camber, blade span, rotor solidity, blade planform and blade kinematics (Kim et al. 2003; Hwang et al. 2008; Benedict et al. 2010b; Benedict et al. 2010a; Benedict et al. 2013). Two important effects that also need to be considered in the design of cycloidal rotors are the virtual camber effect and the blade deflection due to the large radial loads (Kim et al. 2003; Lee et al. 2013; Benedict et al. 2012). In particular, the virtual camber effect occurs because the blades in cyclogyros experience a curvilinear flow and are also oscillating around the blade span axis, thus each point of the blade chord operates at a unique angle of attack and moves with a different local velocity. Because of this, each blade behaves like a cambered airfoil, and exhibits significant deviations in performance with respect to a fixed angle, rectilinear flow configuration.

Computational tools have been widely used for analysing the influence of several fluid-dynamic effects on the aerodynamic behaviour of pitching aerofoils (Wang et al. 2012; Gharali and Johnson 2013), rotary wings (Laxman et al. 2013) and on the efficiency of cyclogyros (Tang et al. 2015). For instance, it was demonstrated, through the usage of numerical tools, that the unsteady interference among blades can significantly alter the flow uniformity on the downstream blades, affecting their aerodynamic performance (Iosilevskii and Levy 2003; Iosilevskii and Levy 2006). Such an interaction may improve the rotor thrust generation by delaying stall on each individual blade (Páscoa and Ilieva 2012; Yu et al. 2013) but it can also reduce their efficiency (Xisto et al. 2014b). The flow on the cyclogyro was also analysed using experimental PIV (Particle Image Velocimetry) techniques, where complex flow patterns were observed, like the formation and convection of the leading edge vortex on the pitching aerofoils (Benedict et al. 2010b), the generation of large regions of rotating flow inside the rotor cage and a skewed exit flow (Benedict et al. 2010a; Nakaie et al. 2010).

For analysing the flow on cycloidal rotors several analytical models have been developed so far. Wheatley (Wheatley 1933) formulated a simplified aerodynamic model for the cyclogyro by neglecting the unsteady aerodynamic effects. Sixty eight years later McNabb (McNabb 2001) took into account the unsteady aerodynamics by using the model of Garrick (Garrick 1936) in his formulation, and validated his work by comparing the results obtained with the experimental data of Wheatley (Wheatley and Windler 1935) and Bosch Aerospace (Boschma 2001). Yun et al. (Yun et al. 2005) proposed an analytical model based on blade element theory and on the streamtube model. In their model a control volume is defined, to which is applied the principle of mass, momentum, and energy conservation. Sirohi et al. (Sirohi et al. 2007) introduced an analytical model able to predict rotor performance, where vertical axis wind turbine theory was adapted into the context of aeronautical propulsion. 


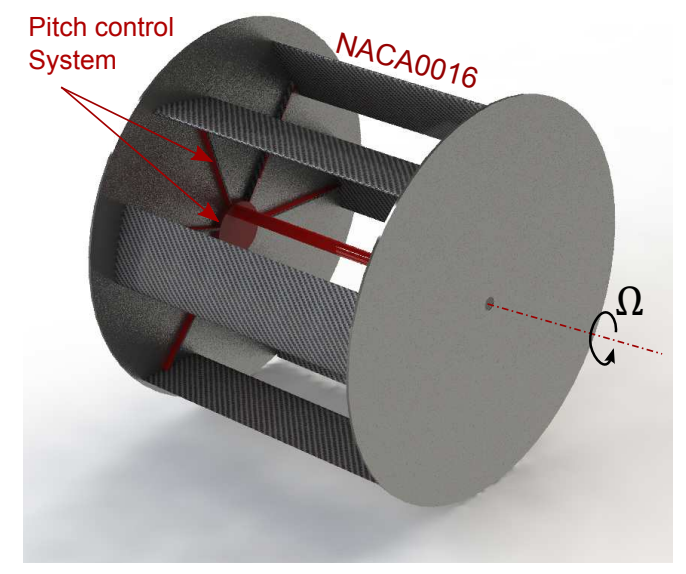

(a)

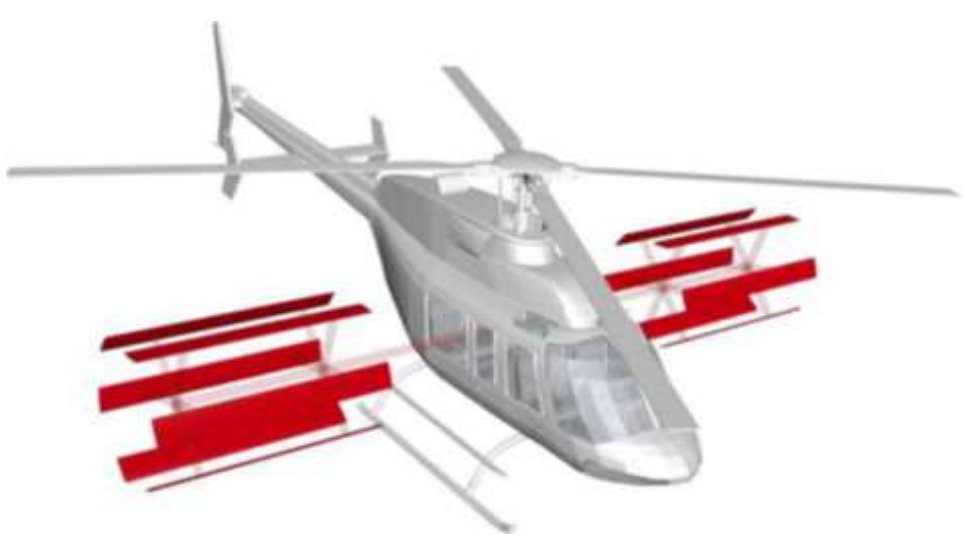

(b)

FIG. 1. a) Three-dimensional representation of a cyclogyro with six NACA0016 blades and a maximum pitch angle of $\mathbf{4 0}$ degree b) Possible incorporation of two cyclogyros into a classical helicopter frame. This compound configuration keeps the main helicopter rotor for generating lift, but can use the two mounted cyclogyros for achieving higher subsonic flight speeds.

In Europe a major multi-country project consortium was set up to develop research on human carrying vehicles propelled by cycloidal rotors. This is the goal of the CROP (Cycloidal Rotor Optimized for Propulsion) project. Part of the work that has been carried out in the frame of the CROP Project (http://www.crop.ubi.pt) is described in this paper. The objectives of the work are to analyze the influence of four key design parameters on the rotor aerodynamic efficiency. An analytical tool was therefore developed for predicting the overall generated thrust and power required by the cyclogyro. Such a model is also able to generate, for a set of geometrical inputs, an instantaneous design and animation of the selected configuration, allowing for a detailed kinematic analysis of the rotor blades and mechanical system. The proposed analytical model was validated with the experimental data for a six-bladed rotor configuration, carried out by the Austrian company IAT21, a partner of the CROP project (Xisto et al. 2014b). A CFD (Computational Fluid Dynamics) procedure was also specifically developed and set up in order to provide a deeper insight in the complex flow patterns that characterize the aerodynamics of a cyclorotor. Both techniques are here employed to study: the effect of the airfoil section (and, in particular, of its thickness); the number of blades (which concurs in the definition of the so-called rotor solidity parameter); the chord-to-radius ratio; and the pitching axis location. It is expected that these four parameters have a strong influence on the overall performance of a large-scale cyclorotor, as those envisaged by the CROP project for potential application to a future generation of manned aerial vehicles.

In the next section an outline of the analytical model formulation will be given. Afterwards, in section 3, the numerical technique will be described, and a cross-comparison between numerical, analytical and experimental results will be brought forward for the purpose of validation. In section 4, results of the parametric study on airfoil thickness, rotor solidity, chord-to-radius ratio and pitching axis location will be presented, and their effect on the performance of the cyclorotor will be discussed.

\section{A SEMI-EMPIRICAL ANALYTICAL MODEL FOR PERFORMANCE ASSESSMENT}

The analytical model employed here represents an improved version of the one presented in (Leger et al. 2015b). The mathematical formulation comprises a kinematic and an aerodynamic 


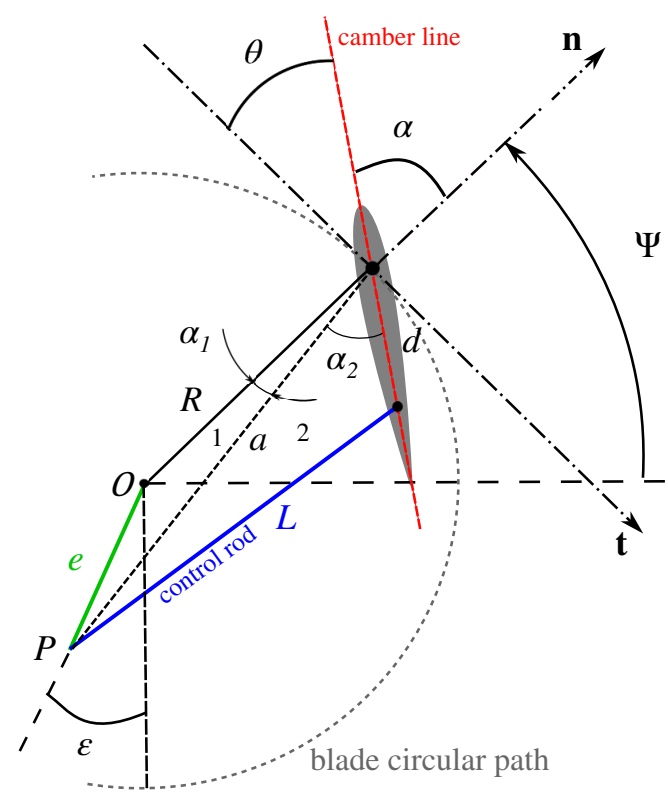

(a)

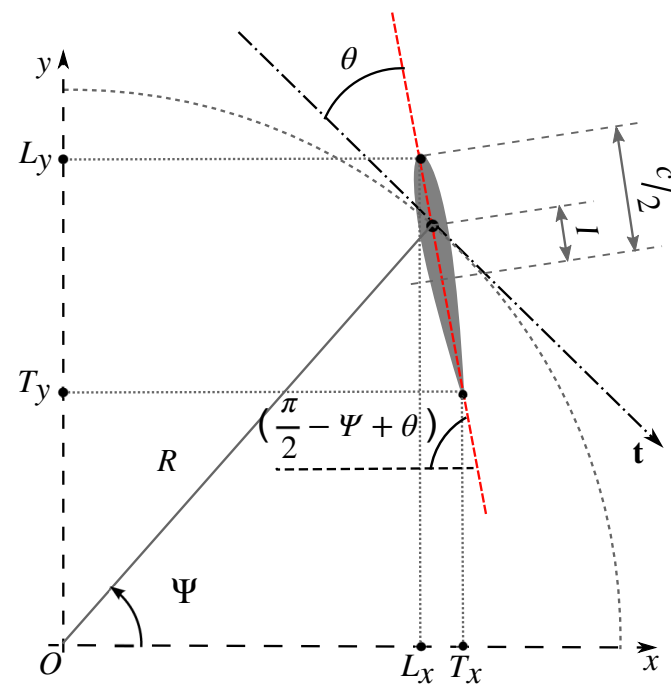

(b)

FIG. 2. a) Kinematic representation of a four-bar-linkage pitching control system. b) Coordinates of the leading and trailing edges.

part. The kinematic description provides the instantaneous position and pitch angle of each blade of the cyclorotor and attests the geometric feasibility of the rotor, a critical aspect that some earlier analytical models did not take into account. The aerodynamic analysis provides an estimate of the generated forces and power requirement of the cyclogyro as a function of its geometric parameters and kinematics, allowing for a quick assessment of the cyclogyro performance.

\section{Kinematic representation}

A schematic representing the kinematics of a single blade of a cycloidal rotor is reported in Fig. 2. The blade rotates with respect to the rotor axis (indicated by the point $\mathrm{O}$ in Fig. 2-a), and is simultaneously capable of pitching around a prescribed axis; as already mentioned, blade pitching endows the cyclorotor with the capability of directing thrust along a given direction. The relative angle $\theta$ between the chord line of the blade and the rotor tangential direction $\mathbf{t}$ is called the pitch angle. The pitch angle varies periodically over a rotation cycle, as a function of the blade azimuthal position $\Psi$.

In existing cyclorotor applications, blade pitching is obtained through the implementation of a specific mechanism; the most widely adopted is the four-bar linkage illustrated in Fig. 2-a. More complex mechanisms, like the three-dimensional cam proposed by Adams et al. (Adams et al. 2013; Adams and Fagley 2013) can also be envisaged, whose analysis is however beyond the scope of the present study. Focusing on the four-bar linkage, by looking at Fig. 2-a), one can observe that the pitch angle is $\theta=\frac{\pi}{2}-\alpha$ with $\alpha=\alpha_{1}+\alpha_{2}$. The distance $a$ from the pivot point (i.e. the intersection between the blade transverse plane and the pitching axis) to the eccentricity point $(P)$, the angle $\alpha_{1}$ and the length $L$ of the control rod, can then be easily represented through simple trigonometric expressions:

$$
\begin{gathered}
a^{2}=e^{2}+R^{2}-2 e R \cos \left(\Psi+\varepsilon+\frac{\pi}{2}\right), \\
\frac{\sin \left(\alpha_{1}\right)}{e}=\frac{\cos \left(\Psi+\varepsilon+\frac{\pi}{2}\right)}{a},
\end{gathered}
$$




$$
L^{2}=a^{2}+d^{2}-2 a d \cos \left(\alpha_{2}\right),
$$

where $R$ is the rotor radius. By combining the previous expressions one can obtain the instantaneous blade pitch angle:

$$
\theta=\frac{\pi}{2}-\sin ^{-1}\left[\frac{e}{a} \cos (\Psi+\varepsilon)\right]-\cos ^{-1}\left[\frac{a^{2}+d^{2}-L^{2}}{2 a d}\right]
$$

From Eqn. 4 it is possible to verify that, for each azimuthal rotor blade position $\Psi$, the pitch angle profile varies just by changing the phase angle of eccentricity $\varepsilon$ and its magnitude $e$, since the length of the control rod $L$ and the distance $d$ are constant for a given mechanical system.

In order to obtain an expression of the overall vertical and horizontal forces that are generated by the rotor, it is necessary to identify the time-dependent position of each blade. Assuming that the initial azimuthal location $\Psi_{1}(0)$ of the pivot point of one blade is known, the others are easily obtained through an arithmetic progression:

$$
\Psi_{i}(0)=\Psi_{1}(0)+\frac{(i-1) 2 \pi}{N} .
$$

where $N$ is the total number of blades of the rotor. Once the initial arrangement of the blades is defined, their time, $t$ dependent position, $\Psi(t)=\Psi_{i}(0)+\Omega t,(\Omega$ is rotor rotational speed in rad $/ \mathrm{s})$ and their time dependent inclination, $\theta(t)=f[\Psi(t)]$, are readily obtained.

In order to account for the aerodynamic effects due to the pitching motion, it is necessary to compute the oscillating angular velocity $\omega$ :

$$
\omega=\frac{d \theta}{\mathrm{d} t}=\frac{d \theta}{d \Psi} \cdot \frac{d \Psi}{d t}=\frac{d \theta}{d \Psi} \cdot \Omega
$$

and the angular acceleration $a_{c c}$ :

$$
a_{c c}=\frac{d \omega}{d t}=\frac{d \omega}{d \Psi} \cdot \frac{d \Psi}{d t}=\frac{d \omega}{d \Psi} \cdot \Omega
$$

where:

$$
\frac{d \omega}{d \Psi}=\frac{d}{d \Psi}\left[\frac{d \theta}{d \Psi} \cdot \Omega\right]=\Omega \cdot \frac{d^{2} \theta}{d \Psi^{2}}
$$

hence:

$$
a_{c c}=\Omega^{2} \cdot \frac{d^{2} \theta}{d \Psi^{2}} .
$$

The coordinates of the leading and trailing edges of each blade are given by the following expressions (see Fig. 2-b):

$$
\begin{aligned}
& L_{x}=R \cos (\Psi)-\left(\frac{1}{2} c+I\right) \cos \left(\frac{\pi}{2}-\Psi+\theta\right), \\
& L_{y}=R \sin (\Psi)+\left(\frac{1}{2} c+I\right) \sin \left(\frac{\pi}{2}-\Psi+\theta\right), \\
& T_{x}=R \cos (\Psi)+\left(\frac{1}{2} c-I\right) \cos \left(\frac{\pi}{2}-\Psi+\theta\right), \\
& T_{y}=R \sin (\Psi)-\left(\frac{1}{2} c-I\right) \sin \left(\frac{\pi}{2}-\Psi+\theta\right),
\end{aligned}
$$




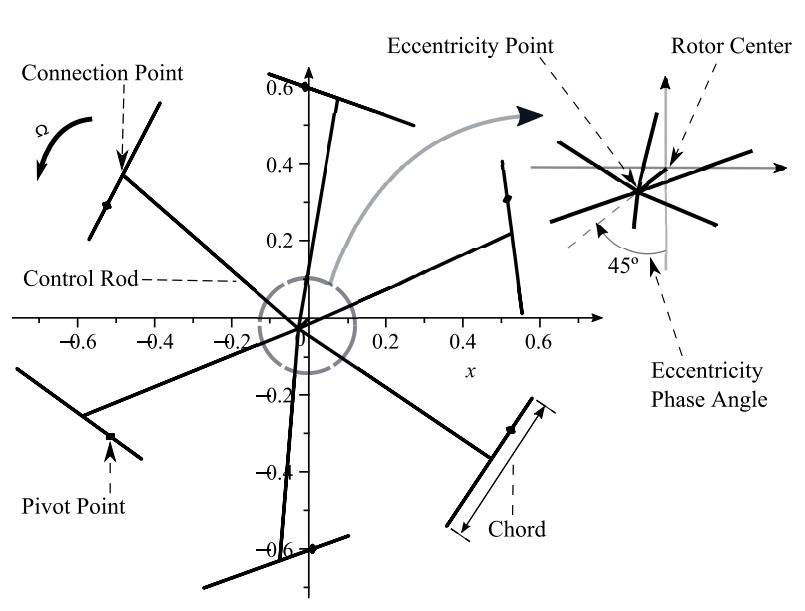

(a)

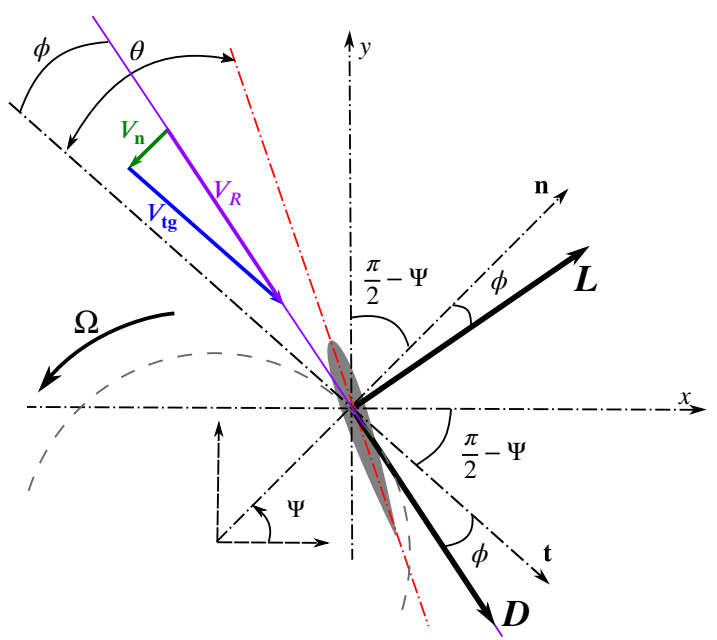

(b)

FIG. 3. a) Instantaneous analytically generated rotor configuration for a pitching amplitude of $\varepsilon=45^{\circ}$. b) Horizontal and vertical components of the resultant force applied to one cyclogyro blade in a pure hovering state.

where $c$ is the chord length and $I$ is the pivot point position measured in a relative rotating and pitching axis. By replacing the rotor blades by their chord line, a simplified representation of the whole rotor geometry as a function of time can be achieved. This can be conveniently translated in an animated sequence by coding the proposed kinematic model in a suitable computer graphics environment. Figure 3-a) shows a snapshot obtained by such a procedure, representing a sixbladed cyclogyro with chord length equal to $0.4 \mathrm{~m}$, rotor diameter equal to $1.2 \mathrm{~m}$, pitch amplitude of $\pm 25^{\circ}$ and a phase angle of eccentricity of $45^{\circ}$.

Using representations such as the one of Fig. 3-a), it is possible to analyze the mechanical feasibility of the cycloidal rotor, for a given set of geometric parameters, which define the rotor dimensions. For instance, it is straightforward to evaluate the geometric interference between blades and the adequacy of the distance between the pivot point and the connection point: as a matter of fact, if the chord is too short there might not be enough space to introduce the control rod.

\section{Aerodynamic modeling}

Figure 3-b) offers a schematic of the aerodynamic forces that are applied to a single cyclogyro blade in a pure hovering state. In such a case, the rotation of the cyclogyro is supposed to induce a nonzero fluid velocity $\mathbf{V}_{I}$, which is generally not aligned with the tangential direction of the blade. Such a velocity adds up to the tangential velocity given to the rotation of the cyclorotor, $\mathbf{V}_{T}=\Omega R \mathbf{t}$. The resultant velocity $\mathbf{V}_{R}$ is given by the vector sum of these two contributions. Its phase angle (called the downwash angle) with respect to the tangential direction $\mathbf{t}$ is indicated as $\phi$ in Fig. 3-b). As a consequence, the actual instantaneous angle of attack of a single blade does not correspond to the pitching angle $\theta$, but to $\theta-\phi$. Consequently, the lift and drag on each blade are necessarily defined along the direction of the vector of the incoming velocity $V_{R}$. By decomposing the lift, $L$, and drag, $D$, vectors, and using a trigonometric angular reduction to the first quadrant, the resultant forces along the $x$ and $y$ directions are defined by the following:

$$
\begin{aligned}
& F_{x}=L \cos (\Psi-\phi)+D \sin (\Psi-\phi), \\
& F_{y}=L \sin (\Psi-\phi)-D \cos (\Psi-\phi) .
\end{aligned}
$$




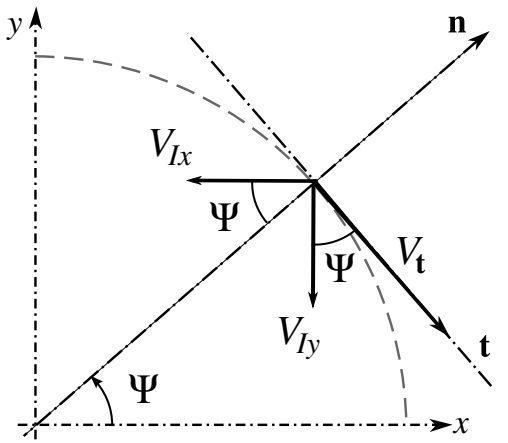

(a)

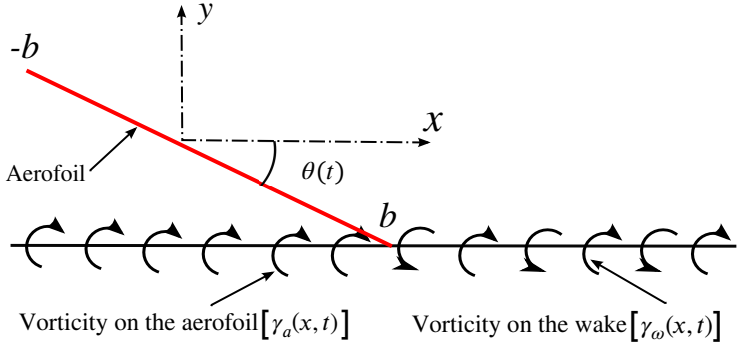

(b)

FIG. 4. Subcomponents of the fluid dynamics module used to analyse the cyclogyro configurations. a) Representation of the induced downwash flow velocity components. b) Vortex linearised model of a pitching flat plate, with a vortex distribution projected on a line parallel to the flow, adapted from

The velocity vector $\mathbf{V}_{R}$ can be decomposed in its tangential and normal directions in order to define the induced downwash angle $\phi=\tan ^{-1}\left(V_{\mathbf{n}} / V_{\mathrm{tg}}\right)$. The tangential and normal components of the resultant velocity are then $V_{\mathrm{tg}}=V_{R} \cos \phi$ and $V_{\mathbf{n}}=V_{R} \sin \phi$, respectively. Alternatively, the resultant velocity can also be expressed through an analysis of the induced downwash velocities, here depicted in Fig. 4-a), where the induced velocity is expressed in terms of its vertical $V_{I y}$ and horizontal $V_{I x}$ components:

$$
\begin{gathered}
V_{\mathrm{tg}}=\Omega R-V_{I x} \sin (\Psi)+V_{I y} \cos (\Psi), \\
V_{\mathbf{n}}=-V_{I x} \cos (\Psi)-V_{I y} \sin (\Psi) .
\end{gathered}
$$

Combining Eqns. (16-17) with Eqns. (14-15), the induced velocities can now be expressed as:

$$
\begin{aligned}
& V_{I x}=\Omega R \sin (\Psi)-V_{R} \sin (\Psi+\phi), \\
& V_{I y}=V_{R} \cos (\Psi+\phi)-V_{\mathrm{t}} \cos (\Psi) .
\end{aligned}
$$

Unsteady aerodynamics, supported on an analytical framework, is still nowadays a direct consequence of the work of Theodorsen on potential flow for the lift and pitching moment on a pitching and plunging thin blade/airfoil (Theodorsen 1935). Such theory considers small perturbations and an harmonic motion. Since the function that Theodorsen found was solely dependent on the reduced frequency of oscillation of the airfoil, $k$, it was concluded that this one was the correct way to consider the flow unsteadiness. In the model proposed here the Garrick (Garrick 1936) model is adopted instead as a starting point. Garrick expanded the early Theodorsen work by introducing an equation for the propulsive force on a pitching thin airfoil. His model is formulated by balancing the conservation of energy in order to compute the propulsive energy as a function of the structural energy and flow wake energy, and also by a direct computation of forces, with the propulsive force computed directly by determining the leading edge suction on a thin airfoil. The model proposed by Garrick will therefore be used to take into account the aerodynamic unsteady effects of the oscillating cyclogyro blades. The two-dimensional unsteady lift is thus given by:

$$
\begin{array}{r}
\frac{L}{S}=\rho b^{2}\left(V_{R} \pi \omega-\pi b I a_{c c}\right)+ \\
C_{L \alpha 3 D} \rho V_{R} b F\left[V_{R}(\theta+\phi)+b\left(\frac{1}{2}-I\right) \omega\right] \\
+\frac{C_{L \alpha 3 D} \rho V_{R} b G}{\Omega}\left[V_{R} \omega+b\left(\frac{1}{2}-I\right) a_{c c}\right],
\end{array}
$$


where $F$ and $G$ are standard Bessel functions of the first and second kinds of argument $k$. These functions are components of the initial Theodorsen's function used to analytically express the vorticity on a harmonically pitching flat plate (Heerenbrink 2011), $\gamma_{a}(x, t)$ (see Fig. 4-b)), whose expression is given by the following:

$$
i \omega \hat{y}_{a}(x)+U_{\infty} \frac{\partial \hat{y}_{a}(x)}{\partial x}=-\frac{1}{2 \pi} \int_{-b}^{b} \frac{\hat{\gamma}_{a}(\xi)}{x-\xi} d \xi+\frac{i \omega}{2 \pi U_{\infty}} \hat{\Gamma} \int_{b}^{\infty} \frac{e^{i \omega\left(\frac{b-\xi}{U \infty}\right)}}{x-\xi} d \xi
$$

The 3-D lift curve slope of the airfoil is given by (Raymer 1989; Etkin and Reid 1996):

$$
\frac{C_{L \alpha 3 D}}{A_{R}}=\frac{2 \pi}{2+\sqrt{\frac{4 \pi^{2} A_{R}^{2}}{C_{l \alpha}^{2}}\left(1+\frac{\tan ^{2}(\Lambda)}{\beta^{2}}\right)+4}}
$$

where $\beta=\sqrt{1-\mathrm{Ma}^{2}}$ is the Prandtl-Glauert compressibility factor, which in the present case is approximately equal to one since, for a low pitch mechanical system, the tip blade speed is generally much lower than the speed of sound, resulting in a very low Mach number. The airfoil sweep angle, $\Lambda$, is neglected since the cyclogyro is composed by straight blades. In this way, the equation for the 3-D lift curve slope reduces to:

$$
C_{L \alpha 3 D}=\frac{2 \pi A_{R}}{2+\sqrt{\frac{4 \pi^{2} A_{R}^{2}}{C_{l \alpha}^{2}}+4}}
$$

The torque, $M$, and power, $P$, are then expressed by the following:

$$
\begin{gathered}
M=\sum_{i=1}\left(F_{y} R \cos \left(\Psi_{i}\right)+F_{x} R \sin \left(\Psi_{i}\right)\right), \\
P=M \Omega .
\end{gathered}
$$

The flow in cyclogyros is quite complex and impossible to be predicted with pure analytical tools. Leger et al. (Leger et al. 2015b) proposed and validated a semi-empirical approach in order to simplify the problem. Such approach required the introduction of an empirical parameter in order to scale the resultant velocity, while neglecting the induced angle $(\phi=0)$ and downwash velocity:

$$
V_{R}=\frac{\Omega R}{E},
$$

where,

$$
E=1.864\left[\frac{34}{N \cdot c \cdot R \cdot S \cdot \theta_{m}}\right]^{-0.187}>1
$$

is a generic function obtained trough experimental data available for the Bosch Aerospace cyclogyro (McNabb 2001). The function of Eqn. (27) was here re-formulated in order to account for the variation of the number of blades. Generally, the maximum and minimum pitch angles are symmetric. However, it is perfectly possible to consider rotor configurations with asymmetric pitching by considering the average value of the difference between the maximum and the minimum pitch angle $\theta_{m}$. The maximum and minimum pitch angles can be obtained by computing the derivative of Eqn. (4). 

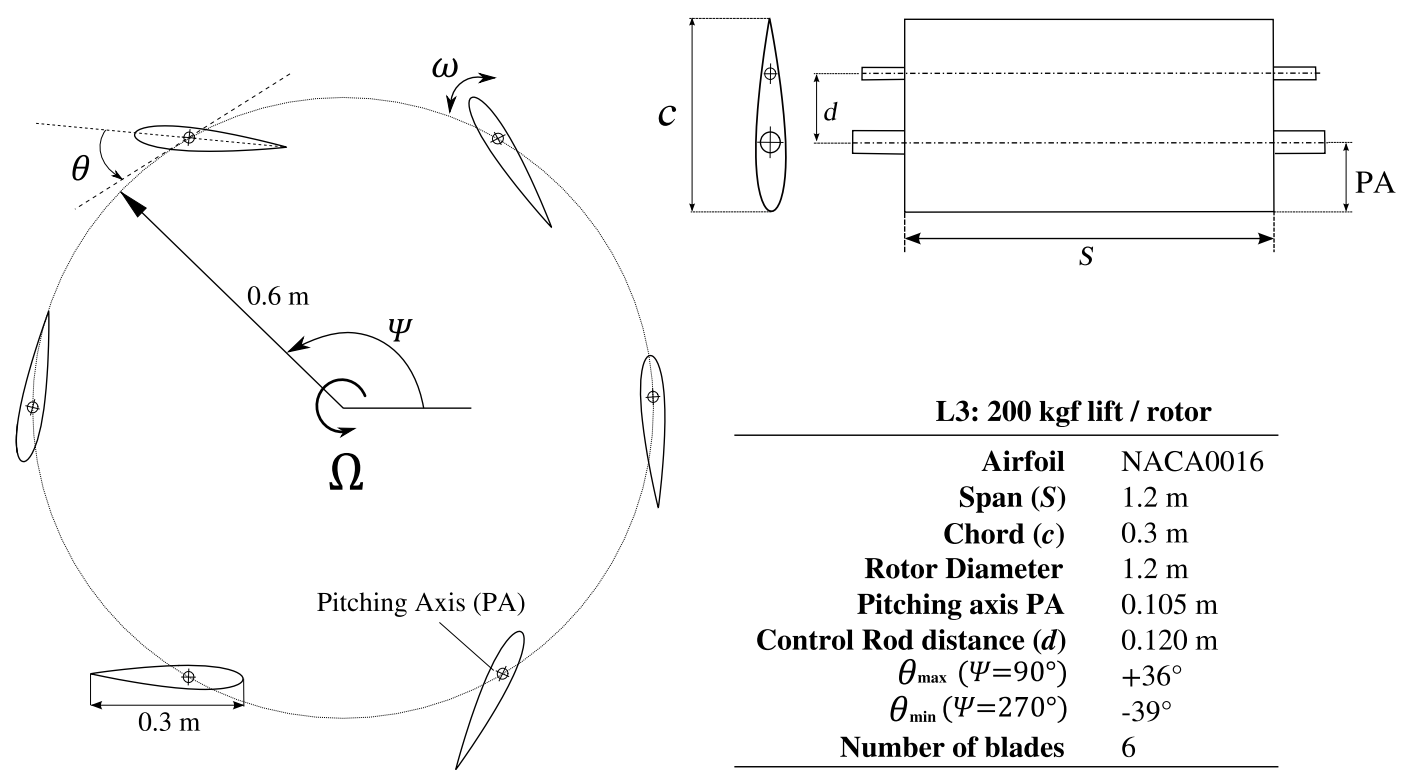

FIG. 5. IAT21 L3 Cycloidal Rotor configuration. The L3-CR comprises six NACA0016 blades with a chord-to-radius ratio equal to 0.5 and a span-to-diameter ratio of one.

\section{NUMERICAL METHOD AND MODEL VALIDATION}

For the purpose of validating the computational and analytical models, the available experimental data for the IAT21 L3-CR configuration (Wills and Schwaiger 2012) is used. The L3-CR comprises six NACA0016 blades with a chord-to-radius ratio equal to 0.5 and a span-to-diameter ratio of one, see Fig. 5. The pitching axis (PA) is located at $35 \%$ of the chord length and the distance between the pitching axis and the control rod is $d=0.120 \mathrm{~m}$. The periodic pitching schedule varies from $\theta=+36^{\circ}$, in the top section of the rotor $\left(\Psi=90^{\circ}\right)$, to $\theta=-39^{\circ}$ in the bottom section of the rotor $\left(\Psi=270^{\circ}\right)$, resulting in an asymmetric pitching profile. This blade pitch angle variation, which is provided by a four-bar-linkage mechanism, can be described by Eqn. (4) and the angular velocity, $\omega$, of the oscillating blades is given by Eqn. (6). The length of the control $\operatorname{rod}(L=0.61 \mathrm{~m})$, the magnitude of eccentricity $(e=0.073 \mathrm{~m})$ and the phase angle of eccentricity $\left(\varepsilon=0^{\circ}\right)$ were defined in order to obtain the desired pitching profile.

The numerical methodology employed in this work was preformed on Ansys Fluent $15^{\circledR}$ (ANSYS 2013). It is based on a Finite-Volume implementation of the incompressible, unsteady Reynoldsaveraged Navier-Stokes equations on 2D, unstructured, moving meshes. The validity incompressibility assumption is later justified on the basis of the maximum velocities involved. For coupling the dependent variables that compose the incompressible Navier-Stokes equations, a pressure-based coupled algorithm is used, which allows performing unsteady computations with a Courant number of order 100 within each time-step. A suitable time accuracy is achieved by employing a fullyimplicit second-order time-discretization method, while, for space interpolation, the second-order linear-upwind differencing scheme is adopted for the convective terms and the central-differences scheme is used in the diffusion terms. For the entire computed range of rotating speeds it is assumed that the compressibility effects are negligible, since the highest blade tip velocity is $V_{T} \approx 63$ $\mathrm{m} / \mathrm{s}$ for a rotating speed of $1000 \mathrm{RPM}$. For such air velocity we obtain a Mach number of $\sim 0.18$, below the threshold of compressible flow $(\mathrm{Ma}=0.3)$. 


\section{Grid and time-step independence study}

A grid sensitivity study was performed in order to reach a final solution that can be consider independent of the mesh size and quality. Three different grids were built with increasing size: grid-0 with $\sim 176,000$ cells; grid-1 with $\sim 300,000$ cells; and grid-2 with $\sim 500,000$ cells. Figure 6 shows one of the numerical grids (grid-1) used for the computation of the IAT21 L3-CR rotor. The overall domain has a circular shape with radius equal to $R_{\infty}=40 R$. A detail view of the rotor mesh and of one of the blades $\left(\Psi=60^{\circ}\right)$ is also reported in Fig. 6 . The outer domain $(\Omega=0$ $\mathrm{rad} / \mathrm{s})$ is a fully unstructured grid that exchanges information with the rotating rotor domain $(\Omega)$, which is also fully unstructured, through a sliding-mesh interface. For the blades six independent circular domains with center located at $35 \%$ of the blade chord length have been generated. These are composed by O-type structured grids in the boundary layer region and unstructured cells for the remaining part of the numerical mesh. The O-type mesh is required in order to achieve a desirable value for the $y^{+}$, in our case less than one. Each one of the domains comprising the blades prescribes the movement that is given by Eqn. 6 and also uses a sliding mesh interface for exchanging information with the rotating rotor. Regarding boundary conditions, for the outer boundary an inlet-outlet condition is imposed and for the walls a non-slip condition for velocity is selected. The time-step used in the grid independence study was $\mathrm{dt} 1=T / 720$, where $T=2 \pi / \Omega$ is the period of pitching and rotation. This time-step size is defined so as to obtain a rotor rotation of $0.5^{\circ}$ per time-step. For all the cases 20 rotor revolutions are computed, although a time-converged periodic solution is found after 10 rotations. Within each time step the solution is computed until the residuals drop bellow $10^{-5}$.

Figure 7-a) shows the thrust variation with rotational speed computed on the three different grids, and using two different turbulence models (Spalart-Almaras, k- $\omega$ SST-Shear Stress Transport). The values for power, computed in the same grids and using the same turbulence models are plotted in Fig. 7-b). For a better comparison the $\log _{10}-\log _{10}$ plots are enlarged in the $\Omega=500 \mathrm{RPM}$ region. One can observe that, as the grid is refined, the computed results for thrust and power do not significantly change. The difference between thrust on grid-1 and grid-2 is less than $1 \%$ and the difference in power for the same grids is less than $0.5 \%$. Therefore one can consider that the results obtained with grid-1 are grid independent. Hence, for the remaining part of this paper, similar grids were generated.

For all the three grids a time-step independence study was also preformed. Figure 7 -c) shows the results obtained for thrust in grid- 1 for three consecutively refined time-steps: dt $0=T / 360$; $\mathrm{dt} 1=T / 720 ; \mathrm{dt} 2=T / 1000$. The results for power, using the same grid and time-steps are plotted in Fig. 7-d). One can observe that there is no significant difference between the plots of dt1 and $\mathrm{dt} 2$, thus following the same conclusions of the grid independence study, the dt1 time-step is used for the remaining part of this paper.

\section{Comparison with experiments}

Figure 8 shows the results obtained using the two different turbulence models (Spalart-Almaras, k- $\omega$ SST) on grid-1 and using the time-step dt1. Both models are reported for providing satisfactory solutions in the presence of adverse pressure gradients and dynamic stall (Martinat et al. 2008). From Fig. 8-a), one can observe that the thrust vs. rotation speed curves, which were computed with the Spalart-Allmaras model, almost overlap the results obtained with k- $\omega$ SST model. Instead, the comparison of the results in terms of rotor power consumption, which are shown in Fig. 8-b), indicates that a better match with experiments is obtained with the Spalart-Allmaras turbulence model. Following these results, we assume that the Spalart-Allmaras model could be used for predicting the overall thrust generated and power consumption in the remaining test cases of this paper. 

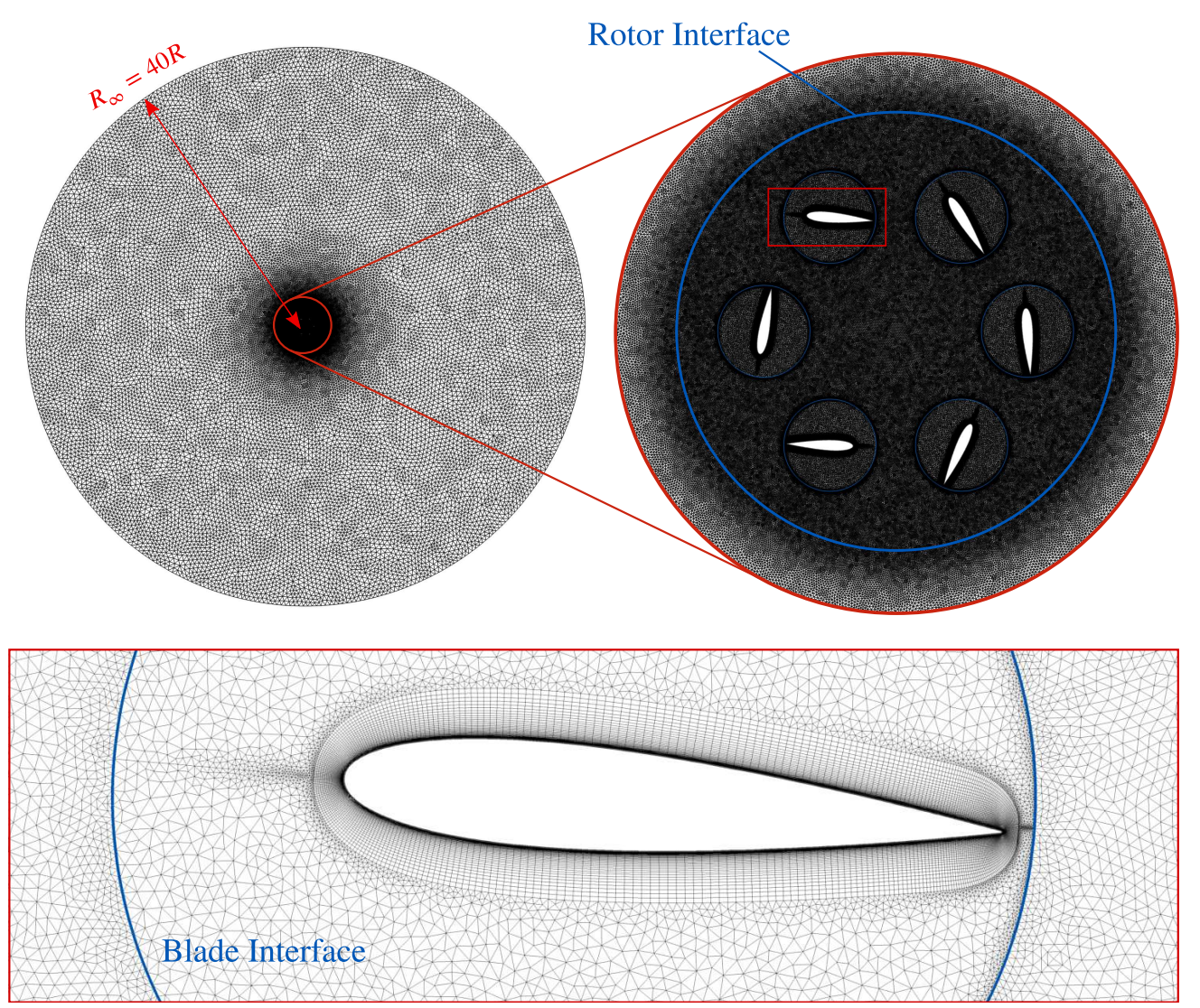

FIG. 6. Two-dimensional numerical domain used for the computation of the IAT21 L3-CR. The overall domain has a circular shape with radius equal to $40 R$.

Figure 8 also draws a comparison between the analytical and CFD results with the experimental data available for the L3-CR configuration. We can observe that the current CFD model over-estimates thrust (Fig. 8-a)) and slightly under-estimates power consumption (Fig. 8-b). It is believed that the over-prediction of thrust is related to the two-dimensional approximation of the rotor, meaning that the 3D flow losses, occurring near the endplate are being neglected (Leger et al. 2015a). The under-estimation of power is also related to the same neglected 3D losses, and also to the fact that only the aerodynamic component of the overall power was computed, meaning that the parasite power, required to rotate the rotor frame (without blades) was not accounted for. A better match is observed in the comparison between analytical results and experimental data. However it should be highlighted that the analytical model was calibrated with a generic linear function $E$ (Eqn. 27), which was defined using the experimental data available from the six-bladed Bosch Aerospace (Boschma 2001), it can be inferred that for cyclogyros working within on-design conditions the present analytical model represents a suitable tool for assessing the rotor efficiency. Nevertheless, it will be shown that in off-design conditions the analytical model fails to predict several important phenomena.

\section{PERFORMANCE PARAMETRIC ANALYSIS}

In the following section a parametric CFD/Analytical analysis of four key parameters on cycloidal rotors was performed. The analysis starts considering the effect of the blade profile, and in particular of the airfoil thickness, on the hovering performance of the rotor. Afterwards, the influence of rotor solidity is addressed, in our case by varying the number of rotor blades and keeping 


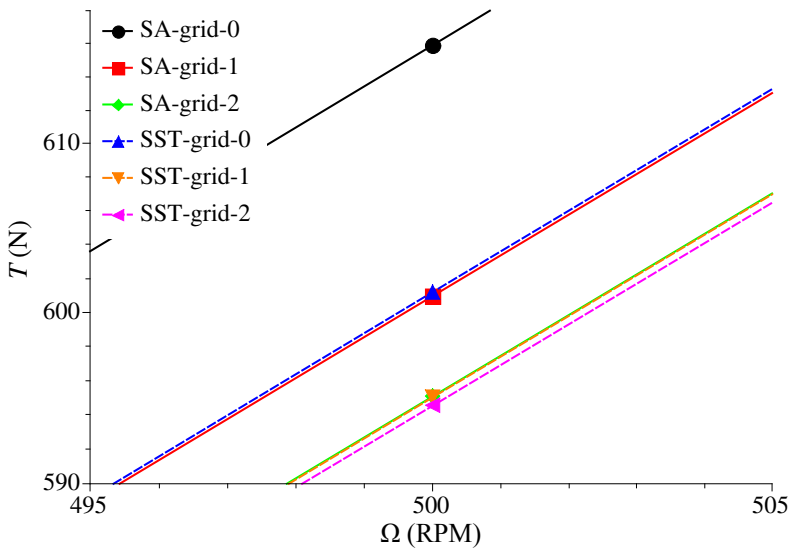

(a) Thrust variation with rotational speed

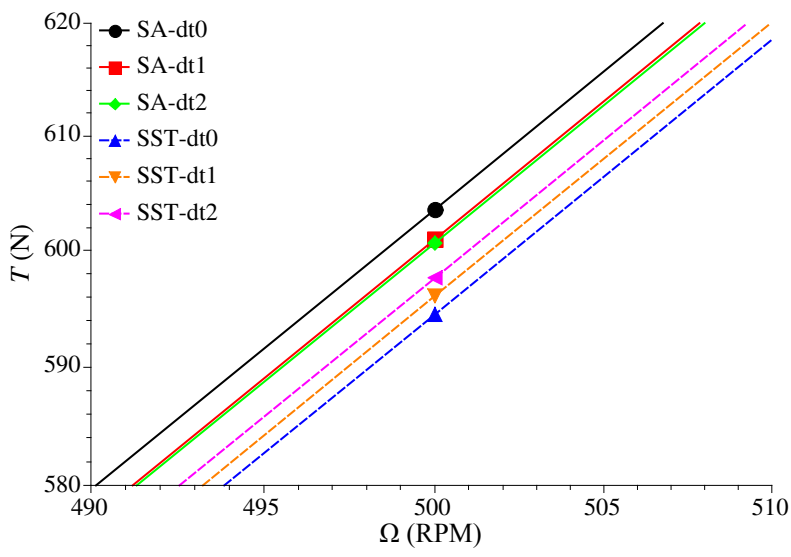

(c) Thrust variation with rotational speed

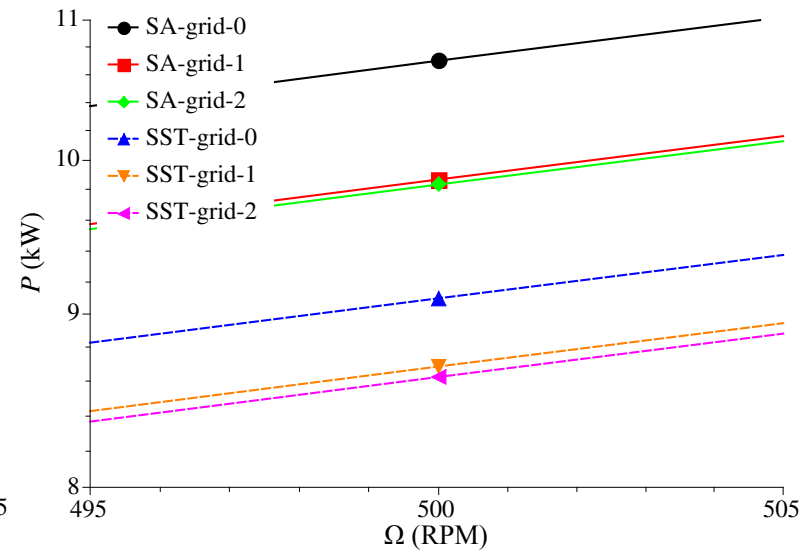

(b) Power variation with rotational speed

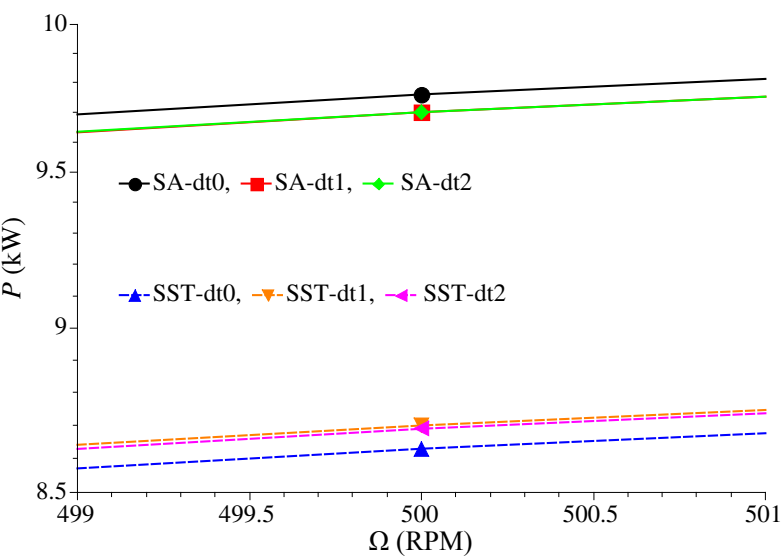

(d) Power variation with rotational speed

FIG. 7. Results obtained for the grid (a-b) and time-step (c-d) independence studies in $\log _{10}-\log _{10}$ plots. The plots are enlarged in the region of $\Omega=500$ RPM.

the chord length constant. In subsection 4, the effect of chord-to-radius ratio (which means again to vary the rotor solidity, this time by keeping the nr. of blades constant) will be studied. The final test comprises the effect of the pitching axis location. In Table 1 the rotor dimensions and the variables used in the parametric study are listed. Throughout the study, the limitations of using analytical models in the prediction of thrust and power in non-ideal (off-design) operating conditions, where more stringent flow patterns occur, will be highlighted.

TABLE 1. Rotor dimensions and variables used in the performance parametric analysis.

\begin{tabular}{|l|l|l|l|}
\hline \multicolumn{2}{|c|}{ Rotor dimensions } & Parameter & Variable \\
\hline Span, $S$ & $1 \mathrm{~m}$ & NACA & $0006-0010-0015-0018$ \\
\hline \multirow{2}{*}{ Radius, $R$} & \multirow{2}{*}{$0.5 \mathrm{~m}$} & Nr. of blades $(N)$ & $2-3-4-6$ \\
\cline { 3 - 4 } & & Chord $(\mathrm{m})$ & $0.125-0.25-0.375$ \\
\hline Pitch amplitude, $\alpha_{0}$ & $40^{\circ}$ & $P A$ location & $12.5 \%-25 \%-35 \%-50 \%$ \\
\hline
\end{tabular}




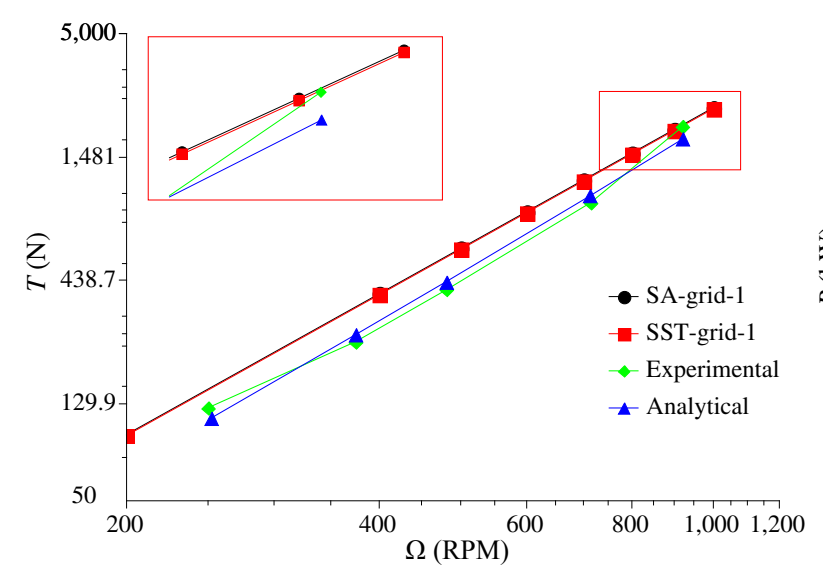

(a)

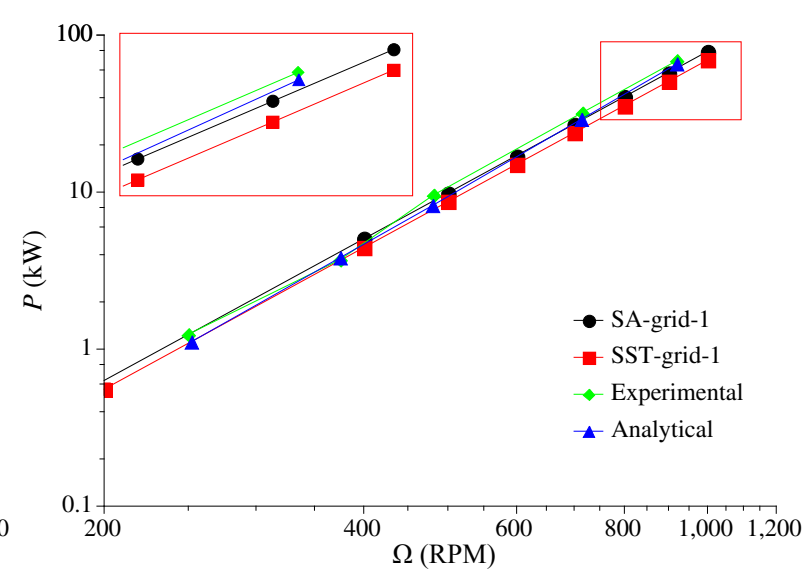

(b)

FIG. 8. Comparison between the analytical and CFD results with experimental data for in $\log _{10}-\log _{10}$ plots: a) Thrust as a function of rotational speed; b) Power as a function of rotational speed.

\section{Influence of cyclogyro blade section geometry}

As the radius is increased, the airfoil section could play a significant role in the aerodynamic performance of the cycloidal rotor. In this section the effect of airfoil thickness for several rotation speeds is analyzed. A similar study was already performed for micro-air-vehicle (MAV) cyclogyros (Benedict et al. 2010b); here such analysis is extended for large scale rotors, which represent the main subject addressed by the CROP FP7 project. The rotor dimensions selected for the analysis are listed in Table 1. In this test case, the number of blades, the chord length and the pitching axis location are fixed parameters, $N=4 ; c=0.25 \mathrm{~m} ; P A=25 \%$, respectively. Four different configurations with increasing airfoil thickness were analyzed, corresponding to the NACA0006; 0010; 0015; 0018 profiles. The pitch angle amplitude was fixed between $40^{\circ}$ and $-40^{\circ}$, and an ideal pitch angle equation was used to described the oscillatory movement of the blades:

$$
\theta=\alpha_{0} \cdot \sin \left(\Omega t+\Psi_{0}\right)
$$

The time derivative of Eq. (28) provides the angular pitching velocity of the blades:

$$
\omega=\dot{\theta}=\alpha_{0} \cdot \Omega \cdot \cos \left(\Omega t+\Psi_{0}\right)
$$

where $\alpha_{0}=40^{\circ}$ is the pitch amplitude and $\Psi_{0}$ is the initial blade position. The rotor rotates counter-clockwise and the pitching axis is located at $25 \%$ of the chord.

The numerical domain is $2 \mathrm{D}$ with a circular shape $\left(R_{\infty}=40 R \mathrm{~m}\right)$. In order to properly solve the boundary layer region an hybrid mesh was again generated with a structured O-type grid near the blades and an unstructured grid in the remaining numerical domain. Each one of the computed cyclogyros is composed by four sub-blocks (blades) that should obey to Eqn. (29). These subblocks communicate with the rotating rotor with a sliding mesh grid interface, and the rotor will also exchange information with the stationary grid with a sliding interface. For the computations, the same solvers and models described in the previous section were employed.

In Fig. 9-a) the power loading ( $P_{L}$, thrust per unit power) vs. disk loading $\left(D_{L}\right.$, thrust per unit area) is shown in a $\log _{10}-\log _{10}$ plot. The disk loading was obtained using the rectangular projected area of the cyclogyro ( $A=S \times 2 R, A=1 \mathrm{~m}^{2}$ in our case). Such a plot is particularly useful, in that it provides a convenient way of measuring the efficiency and the thrust-producing capability 
of the cycloidal rotor (Benedict 2010). As the airfoil thickness is increased, the power loading also increases for all values of disk loading, see Fig. 9-b). Such an effect is given by a simultaneous increase of thrust and a decrease of power. In order to understand the mechanism that is behind the improvement in the rotor efficiency, we have plotted the streamlines for the relative velocity in Fig. 10. We can observe that for the NACA0006 rotor, the flow is separated when each one of the four blades is in the top rotor position $\Psi=90^{\circ}$. As we increase the thickness, the recirculation zone size reduces, until it becomes almost negligible for the NACA0018 test case. This is a direct consequence of the fact that a higher stall angle is associated to thicker aerofoils. We note that, for the tested pitch angle amplitude, the flow only separates in the top position of the cyclogyro, since here the virtual camber effect induces a negative camber on the airfoils.

Virtual camber can introduce significant changes in the aerodynamic behaviour of each blade. Each point along the blade chord line experiences a different local velocity as a consequence of the superimposition of the (steady) rotor angular velocity and of the (harmonic) blade pitch velocity. For the present case, this effect will result in a negative equivalent camber when the blade is in its top position, and a positive equivalent camber when the blade is in its bottom position; such positions coincide with both extremes of the pitching function $\left(90^{\circ}\right.$ and $\left.270^{\circ}\right)$. As a consequence, a larger force is generated by the bottom blade when compared with the top blade of the rotor (negative lift can also be obtained here). It is worth noticing that the virtual camber effect is normally predicted according to the thin airfoil theory undergoing harmonic motion that was proposed by Theodorsen. Such theory only applies to a cyclogyro when the chord-to-radius ratio is small. The virtual camber phenomenon is more pronounced when the blades have a high chord/radius ratio. As a consequence, its actual impact on the performances of the aerofoils needs to be verified for high chord to radius ratio cyclogyros by means of CFD analysis. This will be the subject of subsection 4.

One solution for canceling the virtual camber effect on cyclogyros could be the incorporation of geometrical cambered blades in the rotor. However it is known that in a cyclogyro the blades need to operate at positive and negative angles of attack. This means that if the blade has a positive geometrical camber when it reaches the upper part of the cyclorotor, it will also have a negative camber in the lower half of the cyclorotor, possibly determining a drop on rotor performance due the occurrence of stall. Nevertheless, cambered blades could improve rotor performance if the camber could be changed in the upper and lower half of the rotating cycle. Flow separation could also be reduced, or avoid, with the inclusion of active flow control techniques such as Dielectric Barrier Discharge (DBDs) plasma actuators (Abdollahzadeh et al. 2014b; Abdollahzadeh et al. 2014a; Xisto et al. 2014a; Abdollahzadeh et al. 2015; Xisto et al. 2015).

The analytical (dashed lines) results for power loading as a function of disk loading are also compared in Fig. 9-a) with the CFD results (solid lines). One can observe that a better match between them is obtained as the airfoil thickness increases. This is related to the fact that the analytical model does not take into account the complex flow mechanisms that are associated with flow separation.

\section{Effects of number of blades on cyclogyro operation}

The second parameter we considered is the number of blades. Increasing the number of blades means increasing the solidity parameter, defined as:

$$
\sigma=\frac{N \cdot c}{2 \pi r} .
$$

For this analysis the NACA0018 blade is chosen, as the best performing airfoil among those tested in the previous analysis. The same rotor parameters of the previous test case are kept here, with the exception that now the blade profile is fixed and the number of rotor blades varies. 


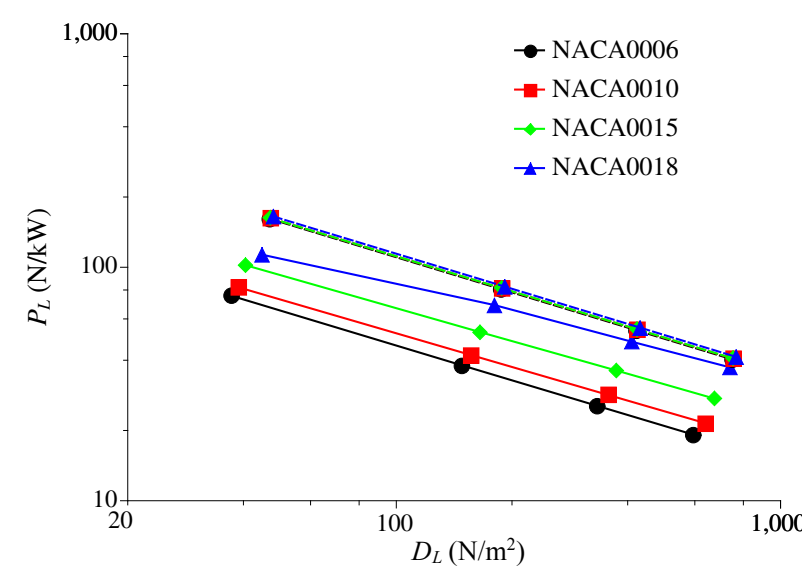

(a)

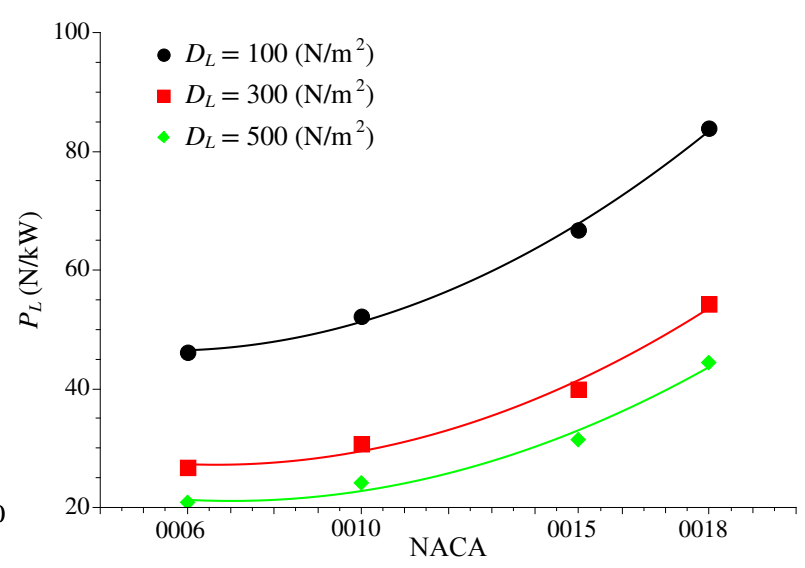

(b)

FIG. 9. Results obtained for the blade profile analysis. a) Power loading vs. disk loading on a $\log _{10}-\log _{10}$ plot: Analytical (dashed lines); CFD (solid lines). b) Power loading variation with airfoil thickness for constant values of disk loading.

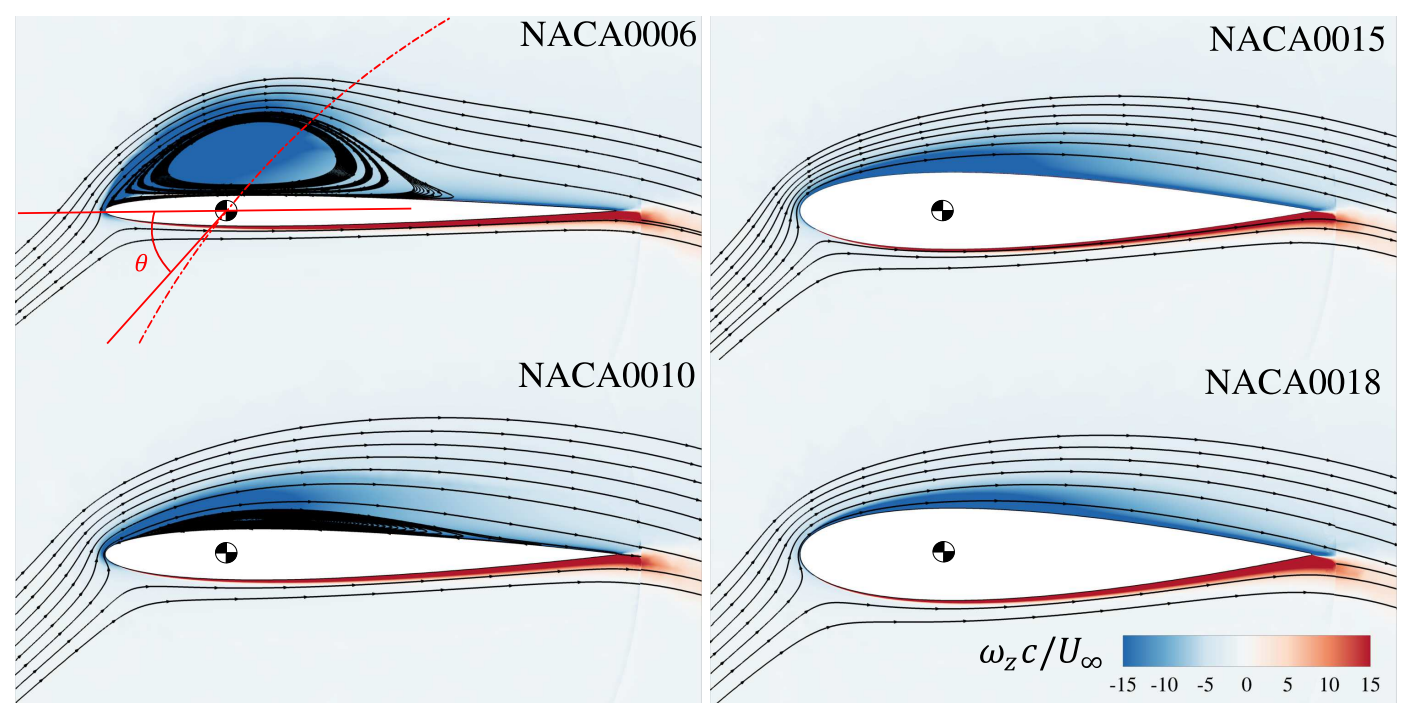

FIG. 10. Streamlines for the relative velocity superimposed on the non-dimensional vorticity contour plots, computed in the rotor azimuthal top position $\left(\Psi=90^{\circ}\right)$.

Figure 11-a) shows the computed (solid lines) and analytically (dash lines) obtained power loading results as a function of disk loading $\left(\log _{10}-\log _{10}\right)$, for a pitch amplitude of $40^{\circ}$. One can see that, in the CFD results, the 3-bladed rotor exhibits the highest values of power loading; therefore, for the selected pitch amplitude, the three-bladed rotor should represent the optimal choice in terms of overall efficiency. However, it is also observed that a higher thrust is achieved by the four-bladed configuration; hence, we can conclude that when power consumption is not a factor, a four-blade configuration is more desirable. When two more blades are added to the rotor ( 6 blades) the interference effects come into play and the rotor power loading, and even the thrust capability, is highly deteriorated. Regarding the analytical results, a good agreement with the CFD data can only be observed, as expected, for configurations with a low number of blades. Once again, under non-ideal conditions, the present analytical model fails to predict thrust and power on 


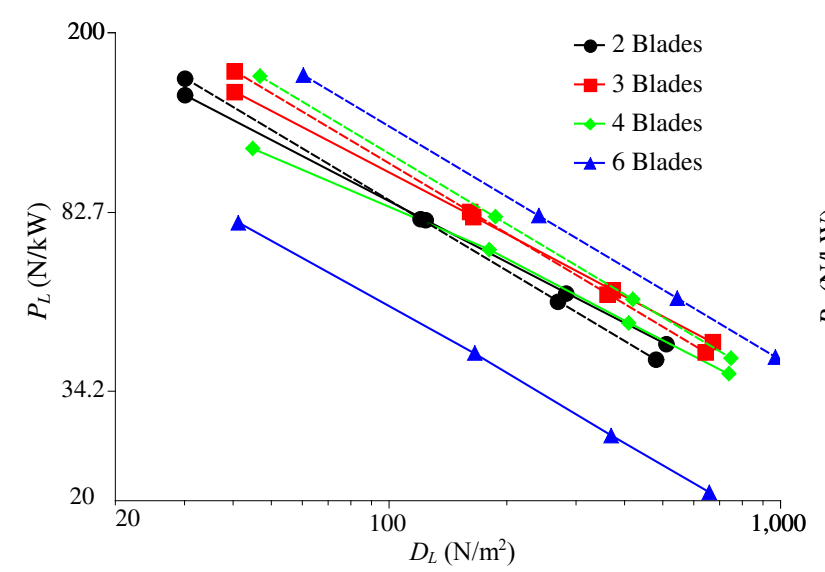

(a)

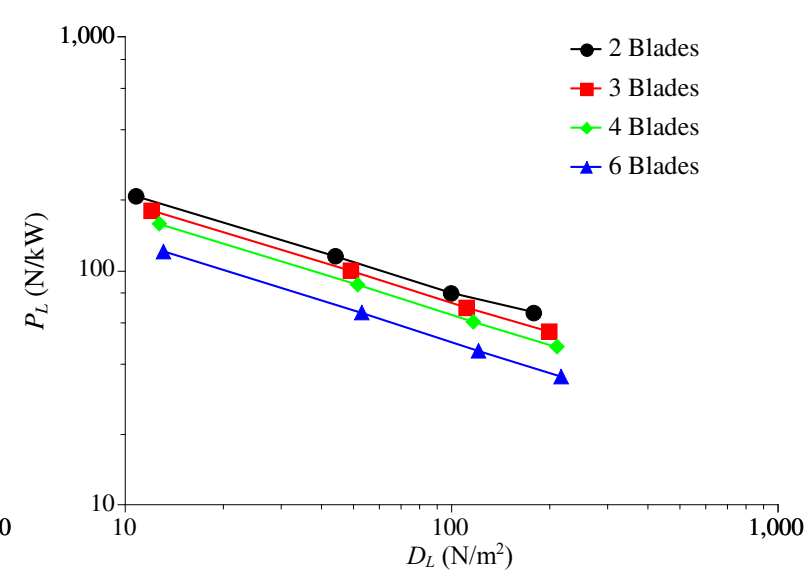

(b)

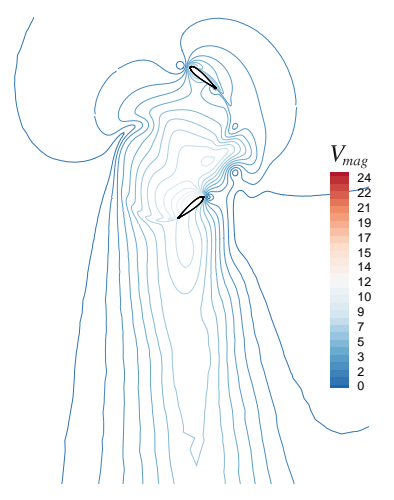

(c)

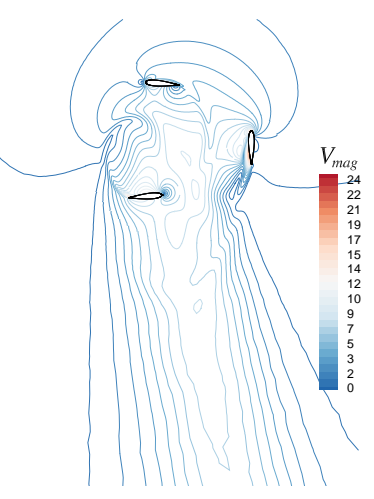

(d)

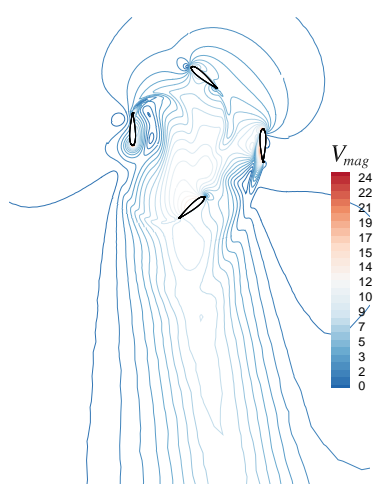

(e)

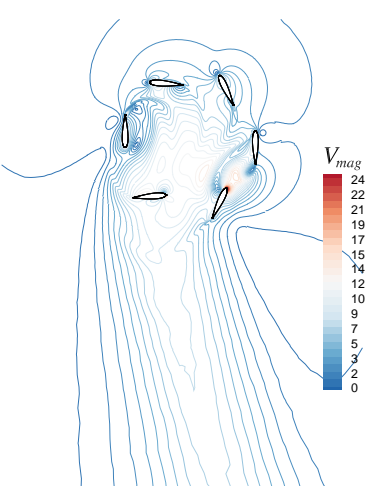

(f)

FIG. 11. Power loading variation with disk loading for rotors with different number of blades on a $\log _{10}-\log _{10}$ plot: Analytical (dashed lines); CFD (solid lines). a) $40^{\circ}$ of pitch amplitude; b) $20^{\circ}$ of pitch amplitude. Velocity magnitude contour plots computed for a $40^{\circ}$ of pitch amplitude at $\Omega=200$ RPM in a rotor with: c) 2 blades; d) 3 blades; e) 4 blades; f) 6 blades.

cyclogyros. This conclusion seems to be in contradiction with the validation test case, where the analytical model preformed better. However, for the parametric study the pitch angle is higher, meaning that the flow separation is more likely to occur.

Figures 11-c) to f) show the velocity magnitude contours for each one of the analysed $40^{\circ}$ pitch configurations. In the same figure it is possible to observe the exit flow direction and magnitude. It was expected that, as the number of blades is increased, the exit flow angle also increases. However the 3-bladed rotor is showing a higher exit flow angle when compared with the 4-bladed rotor test case. A detailed observation of Fig. 11-f) also reveals that the flow is less uniform in the six-bladed rotor when compared with the remaining configurations, following the conclusions of Fig. 11.

A second test case, for a pitch amplitude of $20^{\circ}$, was also performed, in order to verify if the interference effects are also dependent on the pitching amplitude. The power loading vs disk loading variation for the $\alpha_{0}=20^{\circ}$ test cases is shown in Fig. 11-b). In this case, it is the two bladed rotor that exhibits the highest values of power loading for the same disk loading, while the rotor with 6 blades is the one producing the highest thrust, followed by the rotor with 4 blades. This behavior was not observed for the cases at $\alpha_{0}=40^{\circ}$. Regarding power consumption, the six-bladed rotor is seen to remain the most demanding of all. 


\section{Effect of chord length on cyclogyro operation}

In this section three different chord lengths, $0.125,0.25,0.375 \mathrm{~m}$, are tested for a fixed rotor radius and keeping the number of blades at a constant value of $N=4$. In this way it is possible to analyze the effect of chord-to-radius ratio on the cyclogyro efficiency in hovering conditions. For the remaining parameters, the values of the previous sections are used ( $P A=0.25 \%$; NACA0018)

Figure 12-a) shows the power loading variation with disk loading for rotors with different chord lengths, as computed by both the analytical (dashed lines) and CFD (solid lines) models. The analytical model is observed to provide acceptable estimates, with respect to the CFD solution, for a chord-to-radius ratio above 0.5 ; although thrust is being over-estimated for all cases. The biggest discrepancy between the two solutions occurs for $c=0.125 \mathrm{~m}$, as the analytical model is seen to strongly underpredict power consumption.

The results plotted in Fig. 12-a) and b) clearly show that the best chord-to-radius ratio is 0.5 , for this specific configuration; such a finding is in contradiction with the experimental results of Benedict et al. (Benedict et al. 2012) for MAV-scale rotors. They have shown that for a pitch amplitude of $40^{\circ}$, the best $c / R$ is around 0.8 , while here we verify that for $c / R=0.75$ the power loading results are much lower than the ones obtained with $c / R=0.5$. This is a clear indication that, as we up-scale these machines, Reynolds number effects come into play.

Figures 12-c), d), e) show the velocity magnitude contours, which were computed in the three different rotors for a rotating velocity of $200 \mathrm{RPM}$. One can verify that the outflow angle increases with the chord-to-radius ratio; this is mainly is due to the flow curvature effect (virtual camber and incidence), which is more severe in high $c / R$ rotors (Benedict et al. 2012).

\section{Pitching axis location}

The final parameter tested here is the location of the pitching axis (PA) in terms of percentage of chord length. Four locations for the $P A$ are analyzed, namely at $12.5 \% ; 25 \% ; 35 \% ; 50 \%$ of the chord. For this case a chord length of $c=0.25 \mathrm{~m}$ was selected together with a total of four NACA0018 blades.

Figure 13-a) shows the variation of power loading with disk loading for rotors with blades pitching at different axis locations on a $\log _{10}-\log _{10}$ plot. The analytical solution is also plotted (dashed lines). As expected, the analytical model cannot account for the variation of $P A$, providing almost the same results as the PA is moved. Regarding the CFD solution, one can clearly see that the rotors with $P A$ located at $35 \%$ and $50 \%$ of chord length provide the same hover efficiency. Moreover the rotor with $P A$ at $35 \%$ can provide more thrust for the same rotation speed when compared with the other rotors, and the thrust generated by the rotors decreases with the proximity between the $P A$ and the leading edge. This clearly shows that an ideal setup, for the specific configuration of Table 1, corresponds to a $P A$ location between $25 \%$ and $50 \%$ of the chord length.

The CFD results here obtained for large scale cyclogyros are also partially in contrast with the experimental ones obtained by Benedict (Benedict 2010) for MAV-scale rotors. The reduction of power loading as the $P A$ is moved towards the leading edge is confirmed by the experimental findings of Benedict. However, in (Benedict 2010), such a reduction is accompanied by an increase in thrust, which is not detected here. This indicates that the Reynolds number also influences the optimal location of the PA. Figures 13-b), c), d) and e) show the velocity magnitude contour plots that were computed for a rotation speed of $200 \mathrm{RPM}$ for the different $P A$ locations. One can observe that as the $P A$ is moved away form the leading edge, the flow pattern becomes more smooth; this could be an indication that stall effects and pressure drag on the blades are reduced, thus increasing the generation of thrust and decreasing the power consumption.

\section{CONCLUSIONS}

In this paper a novel parametric CFD and analytical study of four key geometrical parameters 


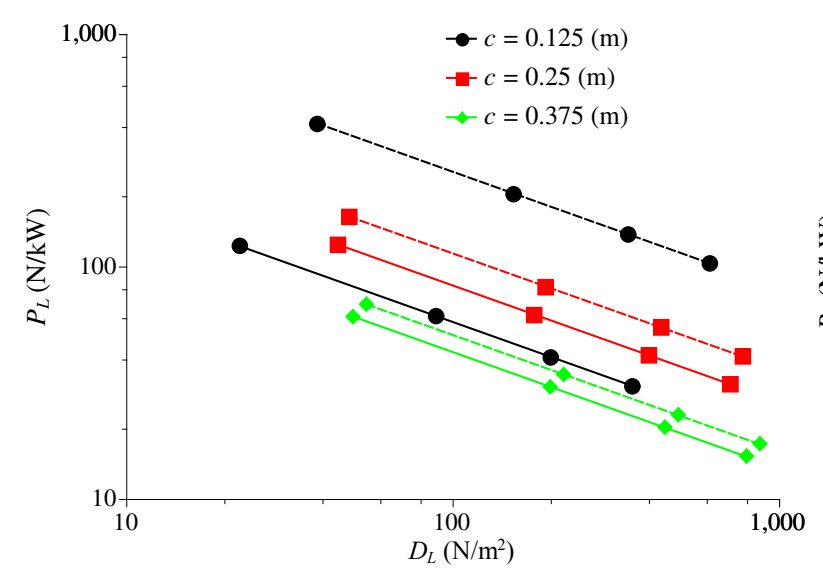

(a)

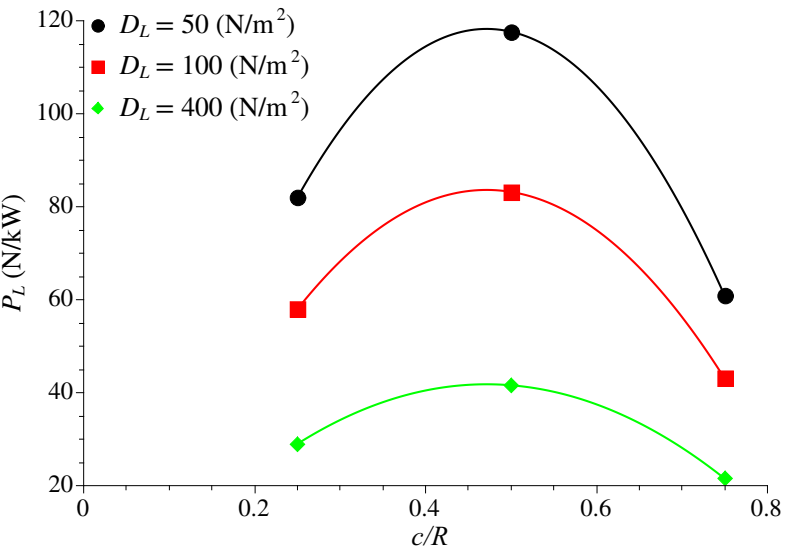

(b)

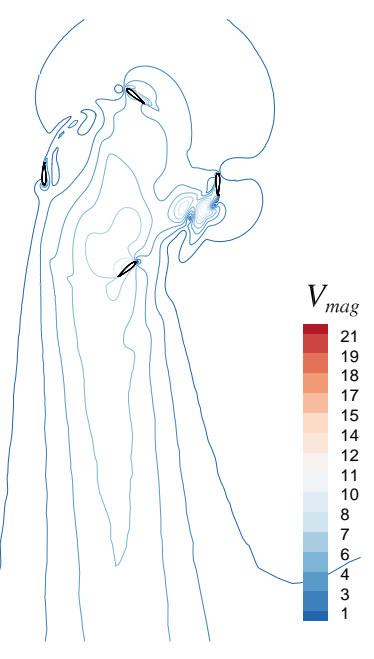

(c)

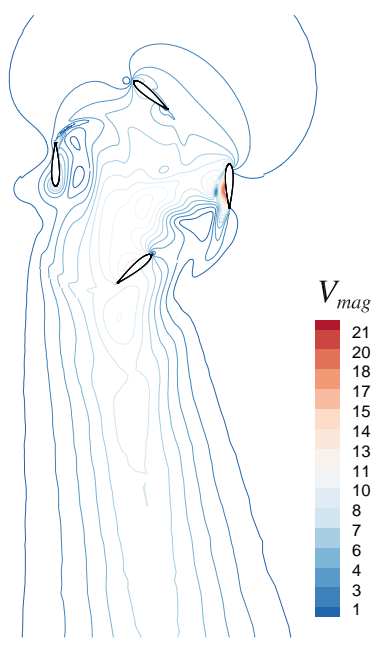

(d)

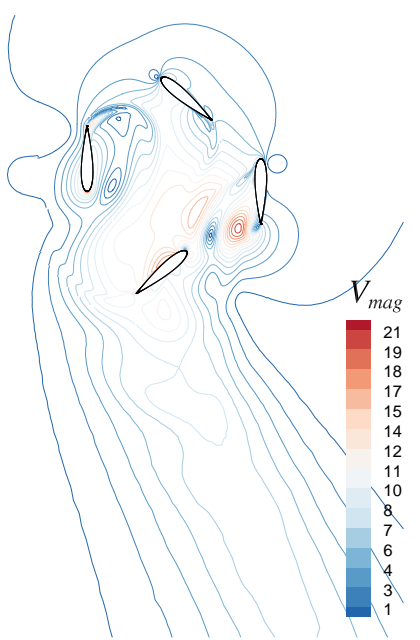

(e)

FIG. 12. a)Power loading variation with disk loading for rotors with different chord-to-radius ratio on a $\log _{10}-\log _{10}$ plot: Analytical (dashed lines); CFD (solid lines). b) Power loading vs. chord-to-radius ratio at a constant disk loading. Velocity magnitude contour plots, computed in a four-bladed rotor for a rotating speed of 200RPM with a varying chord: c) $c=0.125 \mathbf{~ m}$; d) $c=0.25 \mathbf{~ m}$; e) $c=0.375 \mathbf{~ m}$.

ruling the design of cycloidal rotors was performed, namely the blade thickness, the number of blades, the chord-to-radius ratio and the pitching axis location.

For the investigated cases, with fixed pitch amplitude, the rotor efficiency and also thrust capability increases with blade thickness. It should be noted that the efficiency of cyclogyros is also significantly affected by the pitching amplitude. The resultant force increases with the angle of attack until stall is reached at most azimuthal positions. The stall angle can also be increased by increasing the airfoil thickness. However thicker aerofoils tend to increase drag, thus a compromise between lift and drag needs to be obtained for the entire range of operation of the cyclogyro.

Regarding the number of blades, our results show a reduction in efficiency as the number of blades is increased. We have verified that this effect was not observed in MAV scale rotors by other authors (Benedict 2010); however, for similar rotor dimensions $R=0.4 \mathrm{~m}$ (Kim et al. 2003) the 


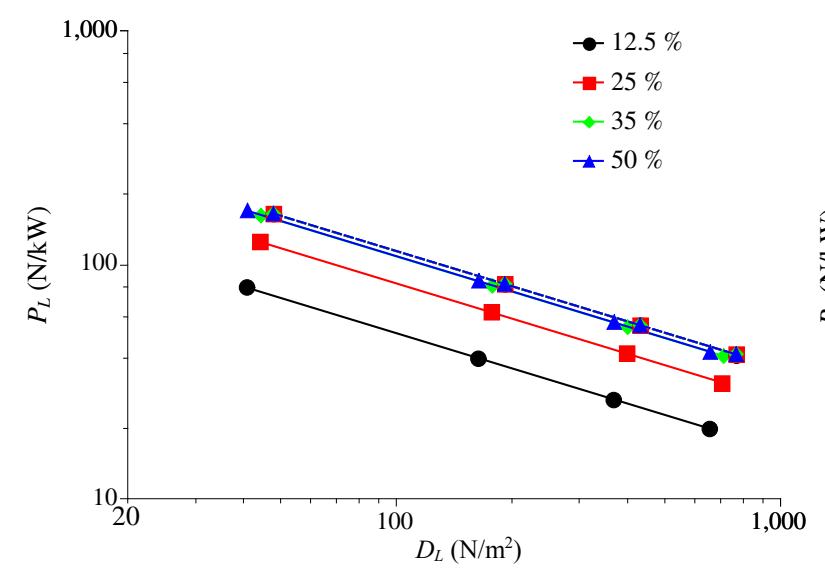

(a)

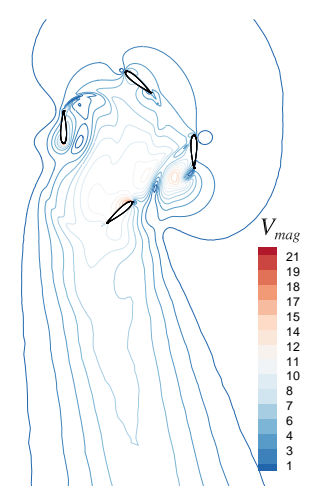

(c)

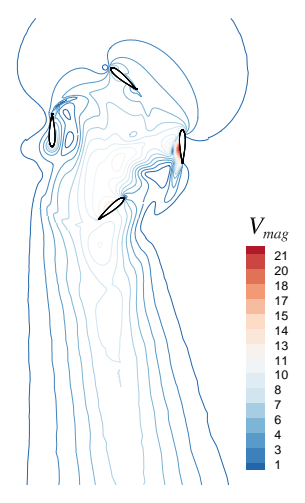

(d)

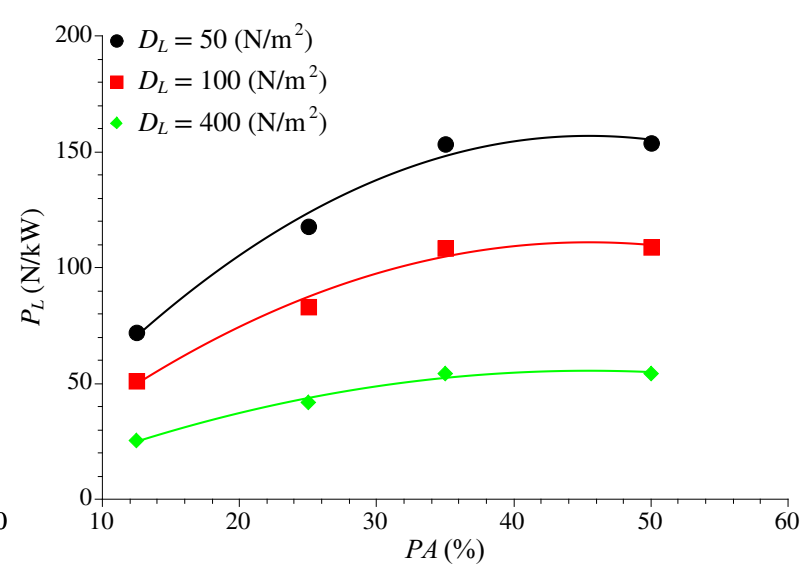

(b)

FIG. 13. a) Power loading variation with disk loading for rotors with different pitching axis on a $\log _{10}-\log _{10}$ plot: Analytical (dashed lines); CFD (solid lines). b) Power loading vs. pitch axis location at a constant disk loading. Velocity magnitude contour plots, computed in a four-bladed rotor for a rotating speed of 200RPM with a varying pitching axis: c) $12.5 \%$ of chord; d) $25 \%$ of chord; e) $35 \%$ of chord; f) $50 \%$ of chord

same trend was observed in experiments. This effect is less pronounced for lower pitch amplitudes, because of a reduction in blade wake.

The CFD results for the chord-to-radius variation have shown that, for this particular rotor, a ratio of $c / R=0.50$ is the one that provides a better hover efficiency. This result contradicts the previous experimental results for MAV-scale cyclogyros operating at a similar pitching amplitude, which indicates that the behaviour of $c / R$ is also a function of the Reynolds number. Such scale effects suggest that, albeit many work has been made at MAV-scale in recent years, it is important to develop research at larger scale to verify the operation and performance of cyclogyros. The results here obtained for the pitching axis location are also in a slight disagreement with the MAV-scale experimental data. The CFD results here obtained for large scale rotors show that the generated thrust is higher for $P A$ of $35 \%$, and decreases as the PA is located closer to the leading edge. This is also an indication that the PA location can also have a strong dependence on the Reynolds number.

The limitations of the proposed analytical model for predicting thrust and power in non-ideal flow conditions were also highlighted. These limitations were evident when mechanisms like flow separation or blade interference effects were of major importance in the performance of cycloidal 
rotors. However, the new analytical model is very useful for the earlier design stages of cyclogyros, in order to define the appropriate rotor dimensions for a specific aircraft payload and mission.

\section{ACKNOWLEDGMENTS}

The present work was performed as part of Project CROP, supported by European Union within the 7th Framework Programme under grant number 323047, and also supported by CMAST, Centre for Mechanical and Aerospace Science and Technology, Research Unit No. 151. 


\section{REFERENCES}

Abdollahzadeh, M., Pascoa, J. C., and Oliveira, P. J. (2014a). "Modified split-potential model for modeling the effect of DBD plasma actuators in high altitude flow control." Current Applied Physics, 14(8), 1160-1170.

Abdollahzadeh, M., Pascoa, J. C., and Oliveira, P. J. (2014b). "Two-dimensional numerical modeling of interaction of micro-shock wave generated by nanosecond plasma actuators and transonic flow." Journal of Computational and Applied Mathematics, 270, 401-416.

Abdollahzadeh, M., Páscoa, J. C., J., O. P., and F., R. (2015). "Numerical design and analysis of a multi-dbd actuator configuration for the experimental testing of acheon nozzle model." Aerospace Science and Technology, in press, 41, 259-273.

Adams, Z., Benedict, M., Hrishikeshavan, V., and Chopra, I. (2013). "Design, Development, and Flight Test of a Small-Scale Cyclogyro UAV Utilizing a Novel Cam-Based Passive Blade Pitching Mechanism." International Journal of Micro Air Vehicles, 5(2), 145-162.

Adams, Z. and Fagley, C. (2013). "Novel Cyclorotor Pitching Mechanism for Operation at Curtate and Prolate Advance Ratios." 51st AIAA Aerospace Sciences Meeting including the New Horizons Forum and Aerospace Exposition, number AIAA2013-0501, Grapevine, Texas, USA (January 710).

ANSYS (2013). Ansys Fluent User's Guide. ANSYS, Inc., Southpointe 275 Technology Drive, Canonsburg, PA 15317.

Benedict, M. (2010). "Fundamental understanding of the cycloidal-rotor concept for micro air vehicle applications." Ph.d. diss., University of Maryland, University of Maryland.

Benedict, M., Jarugumilli, T., and Chopra, I. (2013). "Effect of Rotor Geometry and Blade Kinematics on Cycloidal Rotor Hover Performance." Journal of Aircraft, 50(5), 1340-1352.

Benedict, M., Jarugumilli, T., Lakshminarayan, V., and Chopra, I. (2012). "Experimental and Computational Studies to Understand the Role of Flow Curvature Effects on the Aerodynamic Performance of a MAV-Scale Cycloidal Rotor in Forward Flight." 53rd AIAA/ASME/ASCE/AHS/ASC Structures, Structural Dynamics and Materials Conference, number AIAA2012-1629, Honolulu, Hawaii, USA (April 23-26).

Benedict, M., Ramasamy, M., and Chopra, I. (2010a). "Improving the Aerodynamic Performance of Micro-Air-Vehicle-Scale Cycloidal Rotor: An Experimental Approach." Journal of Aircraft, 47(4), 1117-1125.

Benedict, M., Ramasamy, M., Chopra, I., and Leishman, J. (2010b). "Performance of a Cycloidal Rotor Concept for Micro Air Vehicle Applications." Journal of the American Helicopter Society, $55(2), 022002$.

Boschma, J. (2001). "Modern aviation applications for cycloidal propulsion." 1st AIAA, Aircraft, Technology Integration, and Operations Forum, number AIAA2001-5267, Los Angeles, CA, USA (October 16-18).

Etkin, B. and Reid, L. D. (1996). Dynamics of Flight: Stability and Control, 3rd ed. John Wiley \& Sons.

Foshag, W. F. and Boehler, G. D. (1969). "Review and preliminary evaluation of lifting horizontalaxis rotating-wing aeronautical systems (harwas)." Report No. 69-13, U. S. Army Aviation Materiel Laboratories.

Garrick, I. E. (1936). "Propulsion of a flapping and oscillating airfoil." Report No. 567, National Advisory Committee for Aeronautics.

Gharali, K. and Johnson, D. A. (2013). "Dynamic stall simulation of a pitching airfoil under unsteady freestream velocity." Journal of Fluids and Structures, 42, 228-244.

Gibbens, R. (2003). "Improvements in Airship Control Using Vertical Axis Propellers." AIAA's 3rd 
Annual Aviation Technology, Integration, 83 Operations (ATIO) Forum, number AIAA2003-6853, Denver, Colorado, USA (November 17-19).

Heerenbrink, M. K. (2011). "Simultaneous piv and balance measurements on pitching aerofoil." Msc thesis, Delft University of Technology, Delft University of Technology.

Hwang, S., Min, Y., Lee, H., and Kim, J. (2008). "Development of a Four-Rotor Cyclocopter." Journal of Aircraft, 45(6), 2151-2157.

Ilieva, G., Páscoa, J. C., Dumas, A., and Trancossi, M. (2014). "MAAT - promising innovative design and green propulsive concept for future airship's transport." Aerospace Science and Technology, 35, 1-14.

Iosilevskii, G. and Levy, Y. (2003). "Aerodynamics of the Cyclogiro." 33rd AIAA Fluid Dynamics Conference and Exhibit, number AIAA2003-3473, Orlando, Florida, USA (June 23-26).

Iosilevskii, G. and Levy, Y. (2006). "Experimental and Numerical Study of Cyclogiro Aerodynamics." AIAA Journal, 44(12), 2866-2870.

Kim, S.and Yun, C., Kim, D., Yoon, Y., and Park, I. (2003). "Design and Performance Tests of Cycloidal Propulsion Systems." 44th AIAA/ASME/ASCE/AHS/ASC Structures, Structural Dynamics, and Materials Conference, number AIAA2003-1786, Norfolk, Virigina, USA (April 7-10).

Kirsten, F. (1928). "Cycloidal propulsion applied to aircraft." Transactions of the American Society of Mechanical Engineers, 50, 25-47.

Laxman, V., Venkatesan, C., and Byun, Y. H. (2013). "Influence of blade geometric parameters on aeroelastic response of a helicopter rotor system." Journal of Aerospace Engineering, 26(3), $555-570$.

Lee, H., Min, Y., Kim, S., Lee, W., and Kim, J. (2013). "Design of two-rotored UAV Cyclocopter." 5th Eucass - European Conference for Aerospace Sciences, Munich, Germany (July 1-4).

Leger, J., Páscoa, J., and Xisto, C. (2015a). "3d effects in cyclorotor propulsion systems." Proceedings of the ASME 2015 International Mechanical Engineering Congress 85 Exposition, number IMECE2015-52173, Houston, TX (November 13-19).

Leger, J. A., Páscoa, J. C., and Xisto, C. M. (2015b). "Analytical modeling of a cyclorotor in hovering state." Proceedings of the Institution of Mechanical Engineers Part G Journal of Aerospace Engineering.

Martinat, G., Braza, M., Hoarau, Y., and Harran, G. (2008). "Turbulence modelling of the flow past a pitching \{NACA0012\} airfoil at and reynolds numbers." Journal of Fluids and Structures, 24(8), 1294 - 1303 Unsteady Separated Flows and their Control.

McNabb, M. L. (2001). "Development of a cycloidal propulsion computer model and comparison with experiment." Msc thesis, Mississippi State University, Department of Aerospace Engineering, Mississippi State University, Department of Aerospace Engineering.

Nakaie, Y., Ohta, Y., and Hishida, K. (2010). "Flow measurement around a cycloidal propeller." Journal of Visualization, 13(4), 303-310.

Nakonechny, B. V. (1961). "Experimental performance of a six-bladed vertical axis propeller.." Report No. 1446, Department of the Navy, Hydromechanics Laboratory.

Nozaki, H., Sekiguchi, Y., Matsuuchi, K., Onda, M., Murakami, Y., Sano, M., Akinaga, W., and Fujita, K. (2009). "Research and Development on Cycloidal Propellers for Airships." 18th AIAA Lighter-Than-Air Systems Technology Conference, number AIAA 2009-2850, Seattle, Washington, USA (May 4-7).

Páscoa, J. and Ilieva, G. (2012). "Overcoming stopovers in cycloidal rotor propulsion integration on air vehicles." ASME 2012 International Design Engineering Technical Conferences and Computers and Information in Engineering Conference, number DETC2012-70894, Chicago, Illinois, USA (August 12-15). 
Raymer, D. P. (1989). Aircraft Design: A Conceptual Approach. American Institute of Aeronautics and Astronautics.

Siegel, S., Seidel, J., Cohen, K., and McLaughlin, T. (2007). "A Cycloidal Propeller Using Dynamic Lift." 37th AIAA Fluid Dynamics Conference and Exhibit, number AIAA2007-4232, Miami, FL, USA, 1-14 (June 25-28).

Sirohi, J., Parsons, E., and Chopra, I. (2007). "Hover performance of a cycloidal rotor for a micro air vehicle." Journal of the American Helicopter Society, 52(3), 263-279.

Tang, J., Hu, Y., and Song, B. (2015). "Investigation on the unsteady aerodynamics of cycloidal propeller in hovering flight." Proceedings of the Institution of Mechanical Engineers, Part G: Journal of Aerospace Engineering.

Theodorsen, T. (1935). "General theory of aerodynaniic lnstability and the mechanism of flutter." Report No. 496, NACA.

Trancossi, M., Dumas, A., Xisto, C. M., Páscoa, J. C., and Andrisani, A. (2014). "Roto-cycloid propelled airship dimensioning and energetic equilibrium." SAE 2014 Aerospace System and Technology Conference, Cincinnati, Ohio, USA (September 23-25).

Wang, S., Ingham, D. B., Ma, L., Pourkashanian, M., and Tao, Z. (2012). "Turbulence modeling of deep dynamic stall at relatively low reynolds number." Journal of Fluids and Structures, 33, 191-209.

Wheatley, J. B. (1933). Simplified Aerodynamic Analysis of the Cyclogiro Rotating-Wing System. Technical Notes. Number 467. National Advisory Committee for Aeronautics, Langley Memorial Aeronautical Laboratory (August).

Wheatley, J. B. and Windler, R. (1935). Wind-Tunnel Tests of a Cyclogiro Rotor. Technical Notes. Number 528. National Advisory Committee for Aeronautics, Langley Memorial Aeronautical Laboratory (May).

Wills, D. and Schwaiger, M. (2012). "D-dalus." US EUCOM Science $\&$ Technology Conference, Stuttgart, Germany (June).

Xisto, C. M., Páscoa, J. C., Abdollahzadeh, M., Leger, J. A., Gagnon, L., Masarati, P., Schwaiger, M., and Wills, D. (2015). "Aerodynamic enhancement of cycloidal rotors using dielectric barrier discharge plasma actuators." submited to AIAA Journal of Propulsion and Power.

Xisto, C. M., Páscoa, J. C., Abdollahzadeh, M., Leger, J. A., Schwaiger, M., and Wills, D. (2014a). "PECyT - Plasma Enhaced Cycloidal Thruster." 50th AIAA/ASME/SAE/ASEE Joint Propulsion Conference, Cleveland, OH, USA (July 28-30).

Xisto, C. M., Páscoa, J. C., Leger, J. A., Masarati, P., Quaranta, G., Morandini, M., Gagnon, L., Schwaiger, M., and Wills, D. (2014b). "Numerical modelling of geometrical effects in the performance of a cycloidal rotor." 11th World Conference on Computational Mechanics, number p1848, Barcelona, <http://www.wccm-eccm-ecfd2014.org/admin/files/filePaper/p1848.pdf> (July 20-25).

Yu, H., Bin, L. K., and Wee, T. (2006). "The investigation of cyclogyro design and the Performance." 25th International Congress of Aeronautical Sciences, number 064, Hamburg, Germany (September 3-8).

Yu, H., Fan, D., Gang, W., Chao, W., and Minghang, L. (2013). "Two dimensional numerical simulation of cycloidal propellers with flat plate airfoil in hovering status." AIAA International Powered Lift Conference, number AIAA2013-4244, Los Angeles, CA, USA (August 12-14).

Yun, C. Y., Park, I. K., Hwang, I. S., and Kim, S. J. (2005). "Thrust Control Mechanism of VTOL UAV Cyclocopter with Cycloidal Blades System." Journal of Intelligent Material Systems and Structures, 16(11-12), 937-943. 